\title{
An Index-aware Parametric Model Order Reduction Method for Parametrized Quadratic Differential-Algebraic Equations
}

\author{
Nicodemus Banagaaya ${ }^{\mathrm{a}}$, Peter Benner ${ }^{\mathrm{a}}$, Lihong Feng ${ }^{\mathrm{a}}$, Peter Meuris ${ }^{\mathrm{b}}$, Wim \\ Schoenmaker ${ }^{\mathrm{b}}$ \\ ${ }^{a}$ Max Planck Institute for Dynamics of Complex Technical Systems, Sandtorstr. 1, 39106 Magdeburg, \\ Germany \\ \{banagaaya, benner, feng\}@mpi-magdeburg.mpg.de \\ ${ }^{b}$ Magwel NV, Vital Decosterstraat 44 bus 27, 3000 Leuven, Belgium \\ \{peter.meuris, wim.schoenmaker\}@magwel.com
}

\begin{abstract}
Modeling of sophisticated applications, such as coupled problems arising from nanoelectronics can lead to quadratic differential algebraic equations (DAEs). The quadratic DAEs may also be parametrized, due to variations in material properties, system configurations, etc., and they are usually subject to multi-query tasks, such as optimization, or uncertainty quantification. Model order reduction (MOR), specifically parametric model order reduction (pMOR), is known as a useful tool for accelerating the simulations in a multi-query context. However, pMOR dedicated to this particular structure, has not yet been systematically studied. Directly applying the existing pMOR methods may produce parametric reduced-order models (pROMs) which are less accurate, or may be very difficult to simulate. The same problem was already observed for linear DAEs, and could be eliminated by introducing splitting MOR techniques such as the index-aware MOR (IMOR) methods. We extend the IMOR methods to parameterized quadratic DAEs, thereby producing accurate and easy to simulate index-aware parametric reduced-order models (IpROMs). The proposed approach is so far limited to index-1 one-way coupled problems, but these often appear in computational nanoelectronics. We illustrate the performance of the new approach using industrial models for nanoelectronic structures.
\end{abstract}

Keywords:

model order reduction, quadratic differential-algebraic equations, tractability index

\section{Introduction}

DAEs arise in a variety of applications such as nanoelectronics, electrical networks, gas transport networks, etc, and can be either linear or nonlinear. We consider a special class of nonlinear DAEs in (1) which are quadratic and parameterized by a vector $\mu \in$ 
$\mathbb{R}^{d}$, where $d$ is the number of parameters

$$
\begin{aligned}
\mathbf{E}(\mu) \boldsymbol{x}^{\prime} & =\mathbf{A}(\mu) \boldsymbol{x}+\boldsymbol{x}^{\mathrm{T}} \mathcal{F}(\mu) \boldsymbol{x}+\mathbf{B}(\mu) \boldsymbol{u}, \quad \boldsymbol{x}(0)=\boldsymbol{x}_{0}, \\
\boldsymbol{y} & =\mathbf{C}(\mu) \boldsymbol{x}+\mathbf{D}(\mu) \boldsymbol{u},
\end{aligned}
$$

where $\boldsymbol{x} \in \mathbb{R}^{n}$ is the state vector, the matrix $\mathbf{E}(\mu) \in \mathbb{R}^{n \times n}$ is singular for every parameter vector $\mu$, thus (1) is a system of DAEs. The initial condition $\boldsymbol{x}_{0}$ must be a consistent initial condition, since the initial conditions of DAEs have to satisfy hidden constraints. The system matrices are $\mathbf{A}(\mu) \in \mathbb{R}^{n \times n}, \mathbf{B}(\mu) \in \mathbb{R}^{n \times m}, \mathbf{C}(\mu) \in \mathbb{R}^{\ell \times n}, \mathbf{D}(\mu) \in \mathbb{R}^{\ell \times m}$, and the tensor $\mathcal{F}(\mu)=\left[\mathbf{F}_{1}(\mu)^{\mathrm{T}}, \ldots, \mathbf{F}_{n}(\mu)^{\mathrm{T}}\right]^{\mathrm{T}} \in \mathbb{R}^{n \times n \times n}$ is a 3-D array consisting of $n$ matrices $\mathbf{F}_{i}(\mu) \in \mathbb{R}^{n \times n}$. Each element in $\boldsymbol{x}^{\mathrm{T}} \mathcal{F}(\mu) \boldsymbol{x} \in \mathbb{R}^{n}$ is a scalar $\boldsymbol{x}^{\mathrm{T}} \mathbf{F}_{i}(\mu) \boldsymbol{x} \in \mathbb{R}, i=1, \ldots, n$. The vector $\mu \in \mathbb{R}^{d}$ models the parameter variations. We assume that the matrices $(\mathbf{E}(\mu), \mathbf{A}(\mu), \mathbf{B}(\mu), \mathbf{C}(\mu), \mathbf{D}(\mu))$ and the tensor $\mathcal{F}(\mu)$ have an affine parameter dependence, i.e.,

$$
\mathbf{M}(\mu)=\mathbf{M}_{0}+\sum_{i=1}^{m} f_{i}(\mu) \mathbf{M}_{i},
$$

where the scalar functions $f_{i}$ determine the parameter dependency, which can be nonlinear functions of $\mu$, and $\mathbf{M}_{i}$ can be either a constant matrix or a constant tensor. $\boldsymbol{u}=\boldsymbol{u}(t) \in \mathbb{R}^{m}$ and $\boldsymbol{y}=\boldsymbol{y}(t, \mu) \in \mathbb{R}^{\ell}$ are the inputs (excitations) and the desired outputs (observations), respectively. In order to ensure the uniqueness and existence of the solution of (1), the matrix pencil $\lambda \mathbf{E}(\mu)-\mathbf{A}(\mu)$ must be regular for each parameter $\mu$, where $\lambda \in \mathbb{C}$, i.e., the polynomial $\mathcal{P}(\lambda, \mu)=\operatorname{det}(\lambda \mathbf{E}(\mu)-\mathbf{A}(\mu))$ is not identically zero.

In practice, realistic models have very large dimension $n$ compared to the number of inputs $m$ and desired outputs $\ell$. Despite the ever increasing computational power, simulation of these systems in acceptable time is very difficult, in particular if multiquery tasks are required. MOR aims to reduce this computational burden by generating reduced-order models (ROMs) that are faster and cheaper to simulate, yet accurately represent the original large-scale system behavior [1]. The goal of pMOR is to generate low cost but accurate ROMs that characterize the system response for different values of the parameters. pMOR replaces (1) by a pROM

$$
\begin{aligned}
\mathbf{E}_{r}(\mu) \boldsymbol{x}_{r}{ }^{\prime} & =\mathbf{A}_{r}(\mu) \boldsymbol{x}_{r}+\boldsymbol{x}_{r}^{\mathrm{T}} \mathcal{F}_{r}(\mu) \boldsymbol{x}_{r}+\mathbf{B}_{r}(\mu) \boldsymbol{u}, \quad \boldsymbol{x}_{r}(0)=\boldsymbol{x}_{r 0}, \\
\boldsymbol{y}_{r} & =\mathbf{C}_{r}(\mu) \boldsymbol{x}_{r}+\mathbf{D}_{r}(\mu) \boldsymbol{u},
\end{aligned}
$$

where $\mu$ is symbolically preserved and $\mathbf{E}_{r}(\mu), \mathbf{A}_{r}(\mu) \in \mathbb{R}^{r \times r}, \mathbf{B}_{r}(\mu) \in \mathbb{R}^{r \times m}, \mathbf{C}_{r}(\mu) \in$ $\mathbb{R}^{\ell \times r}, \mathbf{D}_{r}(\mu) \in \mathbb{R}^{\ell \times m}$. The 3D tensor $\mathcal{F}_{r}(\mu)=\left[\mathbf{F}_{r_{1}}^{\mathrm{T}}(\mu), \ldots, \mathbf{F}_{r_{r}}^{\mathrm{T}}(\mu)\right]^{\mathrm{T}} \in \mathbb{R}^{r \times r \times r}$ consists of $r$ matrices $\mathbf{F}_{r_{i}}(\mu) \in \mathbb{R}^{r \times r}$, and $\boldsymbol{x}_{r} \in \mathbb{R}^{r}$ is the reduced state vector of the pROM. The dimension $r \ll n$ of the pROM in (3) is much smaller than that of the original model (1). A good pROM should have small approximation error $\left\|\boldsymbol{y}-\boldsymbol{y}_{r}\right\|$ in a suitable norm $\|\cdot\|$ for 
arbitrary input $\boldsymbol{u}$ and every varying parameter $\mu$. There exist many pMOR techniques which can be used to construct the pROM (3) such as the reduced basis method, Proper Orthogonal Decomposition (POD), the implicit multi-moment-matching method, a survey can be found in [1]. Almost all these methods are based on (Petrov-) Garlerkin projection.

pMOR based on Galerkin projection is done by constructing a projection matrix $\mathbf{V} \in \mathbb{R}^{n \times r}$ so that $\mathbf{V}^{\mathrm{T}} \mathbf{V}=\mathrm{I}$. Then, the pROM of (1) is written in the form

$$
\begin{aligned}
\mathbf{E}_{r}(\mu) \boldsymbol{x}_{r}{ }^{\prime} & =\mathbf{A}_{r}(\mu) \boldsymbol{x}_{r}+\mathbf{V}^{\mathrm{T}}\left[\boldsymbol{x}_{r}^{\mathrm{T}} \tilde{\mathcal{F}}(\mu) \boldsymbol{x}_{r}\right]+\mathbf{B}_{r}(\mu) \boldsymbol{u}, \quad \boldsymbol{x}_{r}(0)=\mathbf{V}^{\mathrm{T}} \boldsymbol{x}_{0}, \\
\boldsymbol{y}_{r} & =\mathbf{C}_{r}(\mu) \boldsymbol{x}_{r}+\mathbf{D}_{r}(\mu) \boldsymbol{u},
\end{aligned}
$$

with the matrices $\mathbf{E}_{r}(\mu)=\mathbf{V}^{\mathrm{T}} \mathbf{E}(\mu) \mathbf{V}, \mathbf{A}_{r}(\mu)=\mathbf{V}^{\mathrm{T}} \mathbf{A}(\mu) \mathbf{V}, \mathbf{B}_{r}(\mu)=\mathbf{V}^{\mathrm{T}} \mathbf{B}(\mu)$, and the tensor $\tilde{\mathcal{F}}=\mathbf{V}^{\mathrm{T}} \mathcal{F}(\mu) \mathbf{V} \in \mathbb{R}^{r \times r \times n}$. In order to project the original consistent initial values onto the initial values for the ROM, the matrix $\mathbf{V}$ is constructed by first transforming (1) into a zero initial condition system, for details see [2]. From, system (4a) we observe that even if the order of the state vector is reduced, the nonlinear part has a computational cost of full dimension $O(n)$. This problem can be eliminated using the fact that $\mathbf{V}^{\mathrm{T}}\left[\boldsymbol{x}_{r}^{\mathrm{T}} \tilde{\mathcal{F}}(\mu) \boldsymbol{x}_{r}\right]$ can be transformed into $\boldsymbol{x}_{r}^{\mathrm{T}} \mathcal{F}(\mu) \boldsymbol{x}_{r}$, where $\mathcal{F}_{r} \in \mathbb{R}^{r \times r \times r}$, using the transformation below.

Proposition 1. Let $\mathbf{W} \in \mathbb{R}^{n \times r}$ be a matrix, $\tilde{\mathcal{F}} \in \mathbb{R}^{r \times r \times n}$ be a $3 \mathrm{D}$ tensor, and $\boldsymbol{x}_{r} \in \mathbb{R}^{r}$, then there exists a $3 \mathrm{D}$ tensor $\mathcal{F}_{r} \in \mathbb{R}^{r \times r \times r}$ such that:

$$
\mathbf{W}^{\mathrm{T}}\left(\boldsymbol{x}_{r}^{\mathrm{T}} \tilde{\mathcal{F}} \boldsymbol{x}_{r}\right)=\boldsymbol{x}_{r}^{\mathrm{T}} \mathcal{F}_{r} \boldsymbol{x}_{r}
$$

Proof. Let $\mathbf{W}=\left[w_{i j}\right] \in \mathbb{R}^{n \times r}$ and $\tilde{\mathcal{F}}=\left[\tilde{\mathbf{F}}_{1}^{\mathrm{T}}, \ldots, \tilde{\mathbf{F}}_{n}^{\mathrm{T}}\right]^{\mathrm{T}}$ with $\tilde{\mathbf{F}}_{i} \in \mathbb{R}^{r \times r}$, then

$$
\begin{aligned}
\mathbf{W}^{\mathrm{T}} \boldsymbol{x}_{r}^{\mathrm{T}} \tilde{\mathcal{F}} \boldsymbol{x}_{r} & =\boldsymbol{x}_{r}^{\mathrm{T}}\left[\sum_{i=1}^{n} \tilde{\mathbf{F}}_{i}^{\mathrm{T}} w_{i 1}, \ldots, \sum_{i=1}^{n} \tilde{\mathbf{F}}_{i}^{\mathrm{T}} w_{i j}, \ldots, \sum_{i=1}^{n} \tilde{\mathbf{F}}_{i}^{\mathrm{T}} w_{i r}\right]^{\mathrm{T}} \boldsymbol{x}_{r}, \\
& =\boldsymbol{x}_{r}^{\mathrm{T}}\left[\mathbf{F}_{r_{1}}^{\mathrm{T}}, \ldots, \mathbf{F}_{r_{r}}^{\mathrm{T}}\right]^{\mathrm{T}} \boldsymbol{x}_{r}, \text { where } \mathbf{F}_{r_{j}}=\sum_{i=1}^{n} w_{i j} \tilde{\mathbf{F}}_{i} \in \mathbb{R}^{r \times r}, j=1, \ldots, r .
\end{aligned}
$$

Hence, $\mathcal{F}_{r}=\left[\mathbf{F}_{r_{1}}^{\mathrm{T}}, \ldots, \mathbf{F}_{r_{r}}^{\mathrm{T}}\right]^{\mathrm{T}} \in \mathbb{R}^{r \times r \times r}$.

The above transformation is numerically feasible, hence, system (4) can be reformulated as in (3). Although existing pMOR methods can be directly applied to parameterized quadratic DAEs, they may produce pROMs which are less accurate or are hard to simulate. This problem used to appear for the case of linear DAEs and it was solved by MOR based on first splitting the DAEs into differential and algebraic subsystems, e.g. the index-aware MOR methods (IMOR) based on admissible projectors [3, 4] and 
balanced truncation model reduction for descriptor systems based on spectral projectors [5]. Applying spectral projectors efficiently to DAEs is described e.g., in the recent survey [6], but this depends on the structure of the singular matrix of the DAE, and may not always be possible. The MOR methods based on splitting techniques always preserve the index structure of the DAEs which guarantees the accuracy of the ROMs.

In this paper, we extend the ideas of the IMOR method proposed in [7] to parameterized quadratic DAEs. We call this method index-aware parameterized model order reduction (IpMOR) for quadratic DAEs. For simplicity, we assume that the index of the system (1) of DAEs is independent of the parameter $\mu$ and its nonlinearity. Thus, its index can be computed using the index concepts for linear DAEs such as the tractability index [7]. For convenience, we shall write $\mathbf{E}=\mathbf{E}(\mu), \mathbf{A}=\mathbf{A}(\mu), \mathbf{B}=\mathbf{B}(\mu), \mathbf{C}=$ $\mathbf{C}(\mu), \mathbf{D}=\mathbf{D}(\mu)$ and $\mathcal{F}=\mathcal{F}(\mu)$, unless otherwise stated. The rest of the paper is organized as follows. In Section 2, we review the decoupling procedure for linear DAEs. Then, in Section 3, we extend the decoupling procedure for linear DAEs to nonlinear quadratic DAEs. Section 4 describes the implicit decoupling of index-1 quadratic DAEs. We introduce the IpMOR for quadratic DAEs in Section 5. Section 6 discusses the construction of projection matrices for pMOR of differential and algebraic subsystems, respectively. Finally, we present some numerical experiments and conclusions.

\section{Decoupling of index-1 linear DAEs}

In this section, we review the decoupling of linear DAEs using projectors and corresponding bases proposed in [3, 4]. Here, we assume $\mathcal{F}=\mathbf{0}$ in (1), thus system (1) can be written as a linear system of DAEs as below

$$
\begin{aligned}
\mathbf{E} \boldsymbol{x}^{\prime} & =\mathbf{A} \boldsymbol{x}+\mathbf{B} \boldsymbol{u}, \quad \boldsymbol{x}(0)=\boldsymbol{x}_{0}, \\
\boldsymbol{y} & =\mathbf{C} \boldsymbol{x}+\mathbf{D} \boldsymbol{u} .
\end{aligned}
$$

It is well known that for linear DAEs of the form (6a), all index concepts (differential, perturbation, strangeness, tractability, etc. ) coincide. Thus the tractability index can be chosen without loss of generality in this case. According to [3], in order to decouple linear DAEs into differential and algebraic parts, we use the definition of the tractability index to construct a matrix and projector chain. A square matrix $\mathbf{Q} \in \mathbb{R}^{n \times n}$ is called projector if and only if $\mathbf{Q}^{2}=\mathbf{Q}$. A projector $\mathbf{Q}$ is called projector onto a subspace $S \subset \mathbb{R}^{n}$ if $S$ is the column space of $\mathbf{Q}$. It is called projector along a subspace $S \subset \mathbb{R}^{n}$ if $S$ is the null space of $\mathbf{Q}$.

Definition 1 (Tractability index [8]). Assume that (6) is solvable, i.e., the matrix pair $(\mathbf{E}, \mathbf{A})$ is regular. We define a matrix and projector chain by setting $\mathbf{E}_{0}:=\mathbf{E}$ and $\mathbf{A}_{0}:=\mathbf{A}$ given by

$$
\mathbf{E}_{j+1}:=\mathbf{E}_{j}-\mathbf{A}_{j} \mathbf{Q}_{j}, \quad \mathbf{A}_{j+1}:=\mathbf{A}_{j} \mathbf{P}_{j}, \quad \text { for } j \geq 0,
$$


where $\mathbf{Q}_{j} \in \mathbb{R}^{n \times n}$ is a projector onto the null space of $\mathbf{E}_{j}$ and $\mathbf{P}_{j}=\mathrm{I}-\mathbf{Q}_{j} \in \mathbb{R}^{n \times n}$. Then there exists an index $\gamma$ such that $\mathbf{E}_{\gamma}$ is nonsingular and all $\mathbf{E}_{j}$ are singular for all $0 \leq j<\gamma-1$. This type of index is called the tractability index.

Next, we use the matrix and projector chain defined in (7) to decouple (6) as follows. For the initial step, we set: $\mathbf{E}_{0}:=\mathbf{E}, \mathbf{A}_{0}:=\mathbf{A}$. Then (6) can be written as

$$
\begin{aligned}
\mathbf{E}_{0} \boldsymbol{x}^{\prime} & =\mathbf{A}_{0} \boldsymbol{x}+\mathbf{B} \boldsymbol{u}, \quad \boldsymbol{x}(0)=\boldsymbol{x}_{0}, \\
\boldsymbol{y} & =\mathbf{C} \boldsymbol{x}+\mathbf{D} \boldsymbol{u} .
\end{aligned}
$$

We then choose the projector $\mathbf{Q}_{0}$ such that it projects onto the null space of $\mathbf{E}_{0}$, and its complementary projector is $\mathbf{P}_{0}:=\mathrm{I}-\mathbf{Q}_{0}$. Using Definition 1 , we can define the matrices, $\mathbf{E}_{1}:=\mathbf{E}_{0}-\mathbf{A}_{0} \mathbf{Q}_{0}, \mathbf{A}_{1}:=\mathbf{A}_{0} \mathbf{P}_{0}$. Then (8) can be written as

$$
\begin{aligned}
\mathbf{E}_{1}\left[\mathbf{P}_{0} \boldsymbol{x}^{\prime}+\mathbf{Q}_{0} \boldsymbol{x}\right] & =\mathbf{A}_{1} \boldsymbol{x}+\mathbf{B} \boldsymbol{u}, \quad \boldsymbol{x}(0)=\boldsymbol{x}_{0}, \\
\boldsymbol{y} & =\mathbf{C}\left[\mathbf{P}_{0} \boldsymbol{x}+\mathbf{Q}_{0} \boldsymbol{x}\right]+\mathbf{D} \boldsymbol{u} .
\end{aligned}
$$

Assume that $\mathbf{E}_{1}$ is nonsingular, then (9) can be written as

$$
\begin{aligned}
\mathbf{P}_{0} \boldsymbol{x}^{\prime}+\mathbf{Q}_{0} \boldsymbol{x} & =\mathbf{E}_{1}^{-1} \mathbf{A}_{1} \boldsymbol{x}+\mathbf{E}_{1}^{-1} \mathbf{B} \boldsymbol{u}, \quad \boldsymbol{x}(0)=\boldsymbol{x}_{0}, \\
\boldsymbol{y} & =\mathbf{C} \mathbf{P}_{0} \boldsymbol{x}+\mathbf{C} \mathbf{Q}_{0} \boldsymbol{x}+\mathbf{D} \boldsymbol{u} .
\end{aligned}
$$

Since $\mathbf{E}_{1}$ is nonsingular, the system of DAEs in (6) is of tractability index-1 or is an index-1 system of DAEs. If we left multiply (10a) by $\mathbf{P}_{0}$ and $\mathbf{Q}_{0}$, separately, we obtain the decoupled equivalent system of (6):

$$
\begin{aligned}
\dot{\boldsymbol{x}}_{P} & =\mathbf{P}_{0} \mathbf{E}_{1}^{-1} \mathbf{A}_{0} \boldsymbol{x}_{P}+\mathbf{P}_{0} \mathbf{E}_{1}^{-1} \mathbf{B} \boldsymbol{u}, \quad \boldsymbol{x}_{P}(0)=\mathbf{P}_{0} \boldsymbol{x}_{0}, \\
\boldsymbol{x}_{Q} & =\mathbf{Q}_{0} \mathbf{E}_{1}^{-1} \mathbf{A}_{0} \boldsymbol{x}_{P}+\mathbf{Q}_{0} \mathbf{E}_{1}^{-1} \mathbf{B} \boldsymbol{u}, \\
\boldsymbol{y} & =\mathbf{C} \boldsymbol{x}_{P}+\mathbf{C} \boldsymbol{x}_{Q}+\mathbf{D} \boldsymbol{u},
\end{aligned}
$$

where $\boldsymbol{x}_{P}:=\mathbf{P}_{0} \boldsymbol{x}$ and $\boldsymbol{x}_{Q}:=\mathbf{Q}_{0} \boldsymbol{x}$. Here (11a) and (11b) are the differential and algebraic parts of system (6), respectively, and the output can be obtained through (11c).

Note that the decoupled system (11) is of dimension $2 n$ while the system (6) is of dimension $n$. This implies that decoupling using projectors does not preserve the dimension of the original system. In [7], a remedy to this problem is proposed by using the linearly independent columns of projectors $\mathbf{Q}_{0}, \mathbf{P}_{0} \in \mathbb{R}^{n \times n}$. Let $n_{q}$ be the dimension of the null space of $\mathbf{E}_{0}$, and $n_{p}=n-n_{q}$. Define matrices $\mathbf{q}_{0} \in \mathbb{R}^{n \times n_{q}}$ and $\mathbf{p}_{0} \in \mathbb{R}^{n \times n_{p}}$ whose columns are linearly independent and span the column spaces of $\mathbf{Q}_{0}$ and $\mathbf{P}_{0}$, respectively. Then, we can build an invertible matrix $\left(\mathbf{p}_{0}, \mathbf{q}_{0}\right) \in \mathbb{R}^{n \times n}$, whose linearly independent columns form a basis of $\mathbb{R}^{n}$. The state vector $\boldsymbol{x}$ can then be represented with respect to the column 
vectors of $\mathbf{p}_{0}, \mathbf{q}_{0}$ as $\boldsymbol{x}=\mathbf{q}_{0} \xi_{q}+\mathbf{p}_{0} \xi_{p}, \xi_{q} \in \mathbb{R}^{n_{q}}, \xi_{p} \in \mathbb{R}^{n_{p}}$, which implies that $\boldsymbol{x}_{P}=\mathbf{p}_{0} \xi_{p}$ and $\boldsymbol{x}_{Q}=\mathbf{q}_{0} \xi_{q}$.

Let the inverse $\left(\mathbf{p}_{0}, \mathbf{q}_{0}\right)^{-1}$ be defined and partitioned as $\left(\mathbf{p}_{0}, \mathbf{q}_{0}\right)^{-1}:=\left(\mathbf{p}_{0}^{*}, \mathbf{q}_{0}^{*}\right)$, where $\mathbf{p}_{0}^{*} \in \mathbb{R}^{n \times n_{p}}, \mathbf{q}_{0}^{*} \in \mathbb{R}^{n \times n_{q}}$, then we have

$$
\mathbf{q}_{0}^{* \mathrm{~T}} \mathbf{q}_{0}=\mathrm{I}_{n_{q}}, \quad \mathbf{q}_{0}^{* \mathrm{~T}} \mathbf{p}_{0}=\mathbf{0}, \quad \mathbf{p}_{0}^{* \mathrm{~T}} \mathbf{q}_{0}=\mathbf{0}, \quad \mathbf{p}_{0}^{* \mathrm{~T}} \mathbf{p}_{0}=\mathrm{I}_{n_{p}} .
$$

Therefore, $\mathbf{q}_{0}^{* \mathrm{~T}}$ and $\mathbf{p}_{0}^{* \mathrm{~T}}$ are actually the left inverses of $\mathbf{q}_{0}$ and $\mathbf{p}_{0}$, respectively. From $\mathbf{q}_{0}^{* \mathrm{~T}} \mathbf{p}_{0}=\mathbf{0}$ and $\mathbf{p}_{0}^{* \mathrm{~T}} \mathbf{q}_{0}=\mathbf{0}$, we obtain

$$
\mathbf{p}_{0}^{* \mathrm{~T}} \mathbf{P}_{0}=\mathbf{p}_{0}^{* \mathrm{~T}}, \quad \mathbf{q}_{0}^{* \mathrm{~T}} \mathbf{Q}_{0}=\mathbf{q}_{0}^{* \mathrm{~T}}
$$

Substituting $\boldsymbol{x}_{P}=\mathbf{p}_{0} \xi_{p}$ and $\quad \boldsymbol{x}_{Q}=\mathbf{q}_{0} \xi_{q}$ into (11), and simplifying using (12) leads to a decoupled system given by

$$
\begin{aligned}
\xi_{p}^{\prime} & =\mathbf{A}_{p} \xi_{p}+\mathbf{B}_{p} \boldsymbol{u}, \quad \xi_{p}(0)=\mathbf{p}_{0}^{* \mathrm{~T}} \boldsymbol{x}_{P}(0), \\
\xi_{q} & =\mathbf{A}_{q} \xi_{p}+\mathbf{B}_{q} \boldsymbol{u}, \\
\boldsymbol{y} & =\mathbf{C}_{p} \xi_{p}+\mathbf{C}_{q} \xi_{q}+\mathbf{D} \boldsymbol{u},
\end{aligned}
$$

where $\mathbf{A}_{p}=\mathbf{p}_{0}^{* \mathrm{~T}} \mathbf{E}_{1}^{-1} \mathbf{A}_{0} \mathbf{p}_{0} \in \mathbb{R}^{n_{p} \times n_{p}}, \mathbf{B}_{p}=\mathbf{p}_{0}^{* \mathrm{~T}} \mathbf{E}_{1}^{-1} \mathbf{B} \in \mathbb{R}^{n_{p} \times m}, \mathbf{A}_{q}=\mathbf{q}_{0}^{* \mathrm{~T}} \mathbf{E}_{1}^{-1} \mathbf{A}_{0} \mathbf{p}_{0} \in$ $\mathbb{R}^{n_{q} \times n_{p}}, \mathbf{B}_{q}=\mathbf{q}_{0}^{* \mathrm{~T}} \mathbf{E}_{1}^{-1} \mathbf{B} \in \mathbb{R}^{n_{q} \times m}, \mathbf{C}_{p}=\mathbf{C p}_{0} \in \mathbb{R}^{\ell \times n_{p}}, \mathbf{C}_{q}=\mathbf{C} \mathbf{q}_{0} \in \mathbb{R}^{\ell \times n_{q}}$. The total dimension of the decoupled system is $n=n_{p}+n_{q}$, hence it preserves the dimension of system (6). In [7], the decoupled system (13) is used to derive the so called index-aware MOR (IMOR) method for index-1 systems.

However, the above decoupling procedure is computationally expensive and needs large storage requirements since it involves the inverse of $\mathbf{E}_{1}$. This motivated the implicit version of the IMOR method proposed in [9], which does not involve $\mathbf{E}_{1}^{-1}$, and is called the implicit-IMOR (IIMOR) method. Its decoupling procedure can be summarized as follows. Instead of using system (10) to decouple the DAE in (6), system (9) can be used which does not involve inversion of $\mathbf{E}_{1}$. Consider another set of matrices $\hat{\mathbf{p}}_{0} \in \mathbb{R}^{n \times n_{p}}$ and $\hat{\mathbf{q}}_{0} \in \mathbb{R}^{n \times n_{q}}$ whose columns are linearly independent and span the null spaces of the matrices $\mathbf{q}_{0}^{\mathrm{T}} \mathbf{A}_{0}^{\mathrm{T}} \in \mathbb{R}^{n_{q} \times n}$ and $\mathbf{E}_{0}^{\mathrm{T}} \in \mathbb{R}^{n \times n}$, respectively. Then (6) can be written into an equivalent decoupled system given by

$$
\begin{aligned}
\mathbf{E}_{p} \xi_{p}^{\prime} & =\mathbf{A}_{p} \xi_{p}+\mathbf{B}_{p} \boldsymbol{u}, \quad \xi_{p}(0)=\mathbf{p}_{0}^{* \mathrm{~T}} \boldsymbol{x}_{P}(0), \\
\mathbf{E}_{q} \xi_{q} & =\mathbf{A}_{q} \xi_{p}+\mathbf{B}_{q} \boldsymbol{u}, \\
\boldsymbol{y} & =\mathbf{C}_{p} \xi_{p}+\mathbf{C}_{q} \xi_{q}+\mathbf{D} \boldsymbol{u},
\end{aligned}
$$

where $\mathbf{E}_{p}=\hat{\mathbf{p}}_{0}^{\mathrm{T}} \mathbf{E}_{0} \mathbf{p}_{0}, \mathbf{A}_{p}=\hat{\mathbf{p}}_{0}^{\mathrm{T}} \mathbf{A}_{0} \mathbf{p}_{0} \in \mathbb{R}^{n_{p} \times n_{p}}, \mathbf{B}_{p}=\hat{\mathbf{p}}_{0}^{\mathrm{T}} \mathbf{B} \in \mathbb{R}^{n_{p} \times m}, \mathbf{E}_{q}=-\hat{\mathbf{q}}_{0}^{\mathrm{T}} \mathbf{A}_{0} \mathbf{q}_{0} \in$ $\mathbb{R}^{n_{q} \times n_{q}}, \mathbf{A}_{q}=\hat{\mathbf{q}}_{0}^{\mathrm{T}} \mathbf{A}_{0} \mathbf{p}_{0} \in \mathbb{R}^{n_{p} \times n_{q}}, \mathbf{B}_{q}=\hat{\mathbf{q}}_{0}^{\mathrm{T}} \mathbf{B} \in \mathbb{R}^{n_{q} \times m}$. We observe that (14) is an implicit 
version of the decoupled system (13) and their solutions must coincide. However, in practice it is computationally cheaper to derive (14) than (13). Both decoupled systems preserve the dimension and the stability of DAEs. System (14) was used to develop the implicit-IMOR method for linear DAEs, see [9]. In the next section, we intend to extend the above decoupling procedures to quadratic nonlinear DAEs.

\section{Decoupling of index-1 quadratic DAEs}

In this section, we extend the implicit and explicit decoupling proposed in [7] and [9], respectively, to quadratic DAEs. Assume system (1) to be of tractability index 1 and its index to be independent of the nonlinearity. Setting $\mathbf{E}_{0}=\mathbf{E}, \mathbf{A}_{0}=\mathbf{A}$ and using the fact that I $=\mathbf{P}_{0}+\mathbf{Q}_{0}$, where $\mathbf{Q}_{0}$ and $\mathbf{P}_{0}$ are projectors as defined in Section 2, then system (1) can be written as

$$
\begin{aligned}
\mathbf{E}_{0}\left[\mathbf{P}_{0}+\mathbf{Q}_{0}\right] \boldsymbol{x}^{\prime} & =\mathbf{A}_{0}\left[\mathbf{P}_{0}+\mathbf{Q}_{0}\right] \boldsymbol{x}+\boldsymbol{x}^{\mathrm{T}}\left[\mathbf{P}_{0}+\mathbf{Q}_{0}\right]^{\mathrm{T}} \mathcal{F}\left[\mathbf{P}_{0}+\mathbf{Q}_{0}\right] \boldsymbol{x}+\mathbf{B} \boldsymbol{u}, \\
\boldsymbol{y} & =\mathbf{C}\left[\mathbf{P}_{0} \boldsymbol{x}+\mathbf{Q}_{0} \boldsymbol{x}\right]+\mathbf{D} \boldsymbol{u},
\end{aligned}
$$

with consistent initial condition $\boldsymbol{x}(0)=\boldsymbol{x}_{0}$. Using Definition 1 , we can define matrices $\mathbf{E}_{1}:=\mathbf{E}_{0}-\mathbf{A}_{0} \mathbf{Q}_{0}, \mathbf{A}_{1}:=\mathbf{A}_{0} \mathbf{P}_{0}$, which satisfy the identities

$$
\mathbf{E}_{1} \mathbf{P}_{0}=\mathbf{E}_{0}, \quad \mathbf{A}_{1}-\mathbf{E}_{1} \mathbf{Q}_{0}=\mathbf{A}_{0} .
$$

Substituting the identities (16) into (15a) and simplifying, we obtain:

$$
\mathbf{E}_{1}\left[\mathbf{P}_{0} \boldsymbol{x}^{\prime}+\mathbf{Q}_{0} \boldsymbol{x}\right]=\mathbf{A}_{1} \boldsymbol{x}+\boldsymbol{x}^{\mathrm{T}}\left[\mathbf{P}_{0}+\mathbf{Q}_{0}\right]^{\mathrm{T}} \mathcal{F}\left[\mathbf{P}_{0}+\mathbf{Q}_{0}\right] \boldsymbol{x}+\mathbf{B} \boldsymbol{u} .
$$

Here, and below, for any two matrices $\mathbf{W} \in \mathbb{R}^{n_{1} \times n}, \mathbf{V} \in \mathbb{R}^{n \times n_{2}}$, we use the notation

$$
\mathbf{W} \mathcal{F V}:=\left[\left(\mathbf{W F}_{1} \mathbf{V}\right)^{\mathrm{T}}, \ldots,\left(\mathbf{W} \mathbf{F}_{n} \mathbf{V}\right)^{\mathrm{T}}\right]^{\mathrm{T}} \in \mathbb{R}^{n_{1} \times n_{2} \times n},
$$

which is a $3 \mathrm{D}$ tensor with $\mathbf{F}_{i} \in \mathbb{R}^{n \times n}, i=1, \ldots, n$. Expanding the nonlinear quadratic part of (17), we obtain

$$
\left[\mathbf{P}_{0}+\mathbf{Q}_{0}\right]^{\mathrm{T}} \mathcal{F}\left[\mathbf{P}_{0}+\mathbf{Q}_{0}\right]=\mathbf{P}_{0}^{\mathrm{T}} \mathcal{F} \mathbf{P}_{0}+\mathbf{P}_{0}^{\mathrm{T}} \mathcal{F} \mathbf{Q}_{0}+\mathbf{Q}_{0}^{\mathrm{T}} \mathcal{F} \mathbf{P}_{0}+\mathbf{Q}_{0}^{\mathrm{T}} \mathcal{F} \mathbf{Q}_{0}
$$

Let $\mathcal{F}_{P}=\mathbf{P}_{0}^{\mathrm{T}} \mathcal{F} \mathbf{P}_{0} \in \mathbb{R}^{n \times n \times n}, \mathcal{F}_{P Q}=\mathbf{P}_{0}^{\mathrm{T}} \mathcal{F} \mathbf{Q}_{0} \in \mathbb{R}^{n \times n \times n}, \mathcal{F}_{Q P}=\mathbf{Q}_{0}^{\mathrm{T}} \mathcal{F} \mathbf{P}_{0} \in \mathbb{R}^{n \times n \times n}, \mathcal{F}_{Q}=$ $\mathbf{Q}_{0}^{\mathrm{T}} \mathcal{F} \mathbf{Q}_{0} \in \mathbb{R}^{n \times n \times n}$, and substitute (18) into (17), we obtain

$$
\mathbf{E}_{1}\left[\mathbf{P}_{0} \boldsymbol{x}^{\prime}+\mathbf{Q}_{0} \boldsymbol{x}\right]=\mathbf{A}_{1} \boldsymbol{x}+\boldsymbol{x}^{\mathrm{T}} \mathcal{F}_{P} \boldsymbol{x}+\boldsymbol{x}^{\mathrm{T}} \mathcal{F}_{Q P} \boldsymbol{x}+\boldsymbol{x}^{\mathrm{T}} \mathcal{F}_{P Q} \boldsymbol{x}+\boldsymbol{x}^{\mathrm{T}} \mathcal{F}_{Q} \boldsymbol{x}+\mathbf{B} \boldsymbol{u}
$$

The matrix $\mathbf{E}_{1}$ is nonsingular due to Definition 1 since we have already assumed that (1) is index-1. Otherwise the iteration continues until a nonsingular matrix $\mathbf{E}_{\mu}$ is obtained. Thus (19) can be written as

$$
\begin{aligned}
\mathbf{P}_{0} \boldsymbol{x}^{\prime}+\mathbf{Q}_{0} \boldsymbol{x}=\mathbf{E}_{1}^{-1} \mathbf{A}_{1} \boldsymbol{x}+ & \mathbf{E}_{1}^{-1} \boldsymbol{x}^{\mathrm{T}} \mathcal{F}_{P} \boldsymbol{x} \\
& +\mathbf{E}_{1}^{-1} \boldsymbol{x}^{\mathrm{T}} \mathcal{F}_{Q P} \boldsymbol{x}+\mathbf{E}_{1}^{-1} \boldsymbol{x}^{\mathrm{T}} \mathcal{F}_{P Q} \boldsymbol{x}+\mathbf{E}_{1}^{-1} \boldsymbol{x}^{\mathrm{T}} \mathcal{F}_{Q} \boldsymbol{x}+\mathbf{E}_{1}^{-1} \mathbf{B} \boldsymbol{u} .
\end{aligned}
$$


Left multiplying (20) by $\mathbf{P}_{0}$ and $\mathbf{Q}_{0}$, respectively, we obtain the differential and algebraic parts of system (1). Let $\boldsymbol{x}_{P}=\mathbf{P}_{0} \boldsymbol{x}$ and $\boldsymbol{x}_{Q}=\mathbf{Q}_{0} \boldsymbol{x}$, then (1) can be written as

$$
\begin{aligned}
& \boldsymbol{x}_{P}^{\prime}=\mathbf{P}_{0} \mathbf{E}_{1}^{-1} \mathbf{A}_{1} \boldsymbol{x}_{P}+ \mathbf{P}_{0} \mathbf{E}_{1}^{-1} \boldsymbol{x}_{P}^{\mathrm{T}} \mathcal{F}_{P} \boldsymbol{x}_{P}+\mathbf{P}_{0} \mathbf{E}_{1}^{-1} \boldsymbol{x}_{Q}^{\mathrm{T}} \mathcal{F}_{Q P} \boldsymbol{x}_{P} \\
&+\mathbf{P}_{0} \mathbf{E}_{1}^{-1} \boldsymbol{x}_{P}^{\mathrm{T}} \mathcal{F}_{P Q} \boldsymbol{x}_{Q}+\mathbf{P}_{0} \mathbf{E}_{1}^{-1} \boldsymbol{x}_{Q}^{\mathrm{T}} \mathcal{F}_{Q} \boldsymbol{x}_{Q}+\mathbf{P}_{0} \mathbf{E}_{1}^{-1} \mathbf{B} \boldsymbol{u}, \\
& \boldsymbol{x}_{Q}=\mathbf{Q}_{0} \mathbf{E}_{1}^{-1} \mathbf{A}_{1} \boldsymbol{x}_{P}+ \mathbf{Q}_{0} \mathbf{E}_{1}^{-1} \boldsymbol{x}_{P}^{\mathrm{T}} \mathcal{F}_{P} \boldsymbol{x}_{P}+\mathbf{Q}_{0} \mathbf{E}_{1}^{-1} \boldsymbol{x}_{Q}^{\mathrm{T}} \mathcal{F}_{Q P} \boldsymbol{x}_{P} \\
&+\mathbf{Q}_{0} \mathbf{E}_{1}^{-1} \boldsymbol{x}_{P}^{\mathrm{T}} \mathcal{F}_{P Q} \boldsymbol{x}_{Q}+\mathbf{Q}_{0} \mathbf{E}_{1}^{-1} \boldsymbol{x}_{Q}^{\mathrm{T}} \mathcal{F}_{Q} \boldsymbol{x}_{Q}+\mathbf{Q}_{0} \mathbf{E}_{1}^{-1} \mathbf{B} \boldsymbol{u}, \\
& \boldsymbol{y}=\mathbf{C} \boldsymbol{x}_{P}+\mathbf{C} \boldsymbol{x}_{Q}+\mathbf{D} \boldsymbol{u},
\end{aligned}
$$

with initial condition $\boldsymbol{x}_{P}(0)=\mathbf{P}_{0} \boldsymbol{x}_{0}$. Inspecting (21), we can observe that there are still cross couplings between the state variables of the differential part (21a) and the algebraic part (21b). For the linear part, we can obtain a complete decoupling if we choose the projector $\mathbf{Q}_{0}$ to be the canonical projector [10] in advance, i.e., it satisfies the condition $\mathbf{Q}_{0}=-\mathbf{Q}_{0} \mathbf{E}_{1}^{-1} \mathbf{A}_{0}$. Then, it can easily be proved that $\mathbf{Q}_{0} \mathbf{E}_{1}^{-1} \mathbf{A}_{1}=0$ [10]. Thus assuming, $\mathbf{Q}_{0}$ is a canonical projector simplifies (21) to

$$
\begin{aligned}
& \boldsymbol{x}_{P}^{\prime}=\mathbf{P}_{0} \mathbf{E}_{1}^{-1} \mathbf{A}_{1} \boldsymbol{x}_{P}+ \mathbf{P}_{0} \mathbf{E}_{1}^{-1} \boldsymbol{x}_{P}^{\mathrm{T}} \mathcal{F}_{P} \boldsymbol{x}_{P}+\mathbf{P}_{0} \mathbf{E}_{1}^{-1} \boldsymbol{x}_{Q}^{\mathrm{T}} \mathcal{F}_{Q P} \boldsymbol{x}_{P} \\
&+\mathbf{P}_{0} \mathbf{E}_{1}^{-1} \boldsymbol{x}_{P}^{\mathrm{T}} \mathcal{F}_{P Q} \boldsymbol{x}_{Q}+\mathbf{P}_{0} \mathbf{E}_{1}^{-1} \boldsymbol{x}_{Q}^{\mathrm{T}} \mathcal{F}_{Q} \boldsymbol{x}_{Q}+\mathbf{P}_{0} \mathbf{E}_{1}^{-1} \mathbf{B} \boldsymbol{u}, \\
& \boldsymbol{x}_{Q}=\mathbf{Q}_{0} \mathbf{E}_{1}^{-1} \boldsymbol{x}_{P}^{\mathrm{T}} \mathcal{F}_{P} \boldsymbol{x}_{P}+ \mathbf{Q}_{0} \mathbf{E}_{1}^{-1} \boldsymbol{x}_{Q}^{\mathrm{T}} \mathcal{F}_{Q P} \boldsymbol{x}_{P}+\mathbf{Q}_{0} \mathbf{E}_{1}^{-1} \boldsymbol{x}_{P}^{\mathrm{T}} \mathcal{F}_{P Q} \boldsymbol{x}_{Q} \\
&+\mathbf{Q}_{0} \mathbf{E}_{1}^{-1} \boldsymbol{x}_{Q}^{\mathrm{T}} \mathcal{F}_{Q} \boldsymbol{x}_{Q}+\mathbf{Q}_{0} \mathbf{E}_{1}^{-1} \mathbf{B} \boldsymbol{u}, \\
& \boldsymbol{y}=\mathbf{C} \boldsymbol{x}_{P}+\mathbf{C} \boldsymbol{x}_{Q}+\mathbf{D} \boldsymbol{u},
\end{aligned}
$$

with initial condition $\boldsymbol{x}_{P}(0)=\mathbf{P}_{0} \boldsymbol{x}_{0}$. Considering (22), we need to make some assumptions on the tensor $\mathcal{F}$ such that we can obtain at least a one-way coupling between the differential and algebraic parts in order to avoid cross-coupling. This can be done as follows.

(i) Coupling from differential to algebraic parts

In order to obtain this coupling, we require the assumption that $\mathcal{F}_{Q P}=0, \mathcal{F}_{P Q}=$ $0, \mathcal{F}_{Q}=0$. Thus (22) simplifies to

$$
\begin{aligned}
\boldsymbol{x}_{P}^{\prime} & =\mathbf{P}_{0} \mathbf{E}_{1}^{-1} \mathbf{A}_{1} \boldsymbol{x}_{P}+\mathbf{P}_{0} \mathbf{E}_{1}^{-1} \boldsymbol{x}_{P}^{\mathrm{T}} \mathcal{F}_{P} \boldsymbol{x}_{P}+\mathbf{P}_{0} \mathbf{E}_{1}^{-1} \mathbf{B} \boldsymbol{u}, \quad \boldsymbol{x}_{P}(0)=\mathbf{P}_{0} \boldsymbol{x}_{0}, \\
\boldsymbol{x}_{Q} & =\mathbf{Q}_{0} \mathbf{E}_{1}^{-1} \boldsymbol{x}_{P}^{\mathrm{T}} \mathcal{F}_{P} \boldsymbol{x}_{P}+\mathbf{Q}_{0} \mathbf{E}_{1}^{-1} \mathbf{B} \boldsymbol{u}, \\
\boldsymbol{y} & =\mathbf{C} \boldsymbol{x}_{P}+\mathbf{C} \boldsymbol{x}_{Q}+\mathbf{D} \boldsymbol{u} .
\end{aligned}
$$

(ii) Coupling from algebraic to differential part

In order to obtain this coupling, we require the assumption that $\mathcal{F}_{Q P}=0, \mathcal{F}_{P Q}=$ $0, \mathcal{F}_{P}=0$. Then (22) simplifies to

$$
\begin{aligned}
\boldsymbol{x}_{Q} & =\mathbf{Q}_{0} \mathbf{E}_{1}^{-1} \boldsymbol{x}_{Q}^{\mathrm{T}} \mathcal{F}_{Q} \boldsymbol{x}_{Q}+\mathbf{Q}_{0} \mathbf{E}_{1}^{-1} \mathbf{B} \boldsymbol{u}, \\
\boldsymbol{x}_{P}^{\prime} & =\mathbf{P}_{0} \mathbf{E}_{1}^{-1} \mathbf{A}_{1} \boldsymbol{x}_{P}+\mathbf{P}_{0} \mathbf{E}_{1}^{-1} \boldsymbol{x}_{Q}^{\mathrm{T}} \mathcal{F}_{Q} \boldsymbol{x}_{Q}+\mathbf{P}_{0} \mathbf{E}_{1}^{-1} \mathbf{B} \boldsymbol{u}, \quad \boldsymbol{x}_{P}(0)=\mathbf{P}_{0} \boldsymbol{x}_{0}, \\
\boldsymbol{y} & =\mathbf{C} \boldsymbol{x}_{P}+\mathbf{C} \boldsymbol{x}_{Q}+\mathbf{D} \boldsymbol{u} .
\end{aligned}
$$


We note the above one-way couplings depend on the structure of the tensor $\mathcal{F}$. One may wonder if such tensors exist in practice, fortunately they do exist in applications such as the electro-thermal coupled models as illustrated in Section 7. We can observe that the above decoupled systems double the dimension of the quadratic DAE (1a). However, we can use the same technique as for the linear case in the previous section to avoid this. If we substitute $\boldsymbol{x}_{P}=\mathbf{p}_{0} \xi_{p}$ and $\boldsymbol{x}_{Q}=\mathbf{q}_{0} \xi_{q}$ into (23) and (24) respectively, then we obtain:

(i) Coupling from differential to algebraic parts

$$
\begin{aligned}
\xi_{p}^{\prime} & =\mathbf{A}_{p} \xi_{p}+\mathbf{p}_{0}^{* \mathrm{~T}} \mathbf{E}_{1}^{-1} \xi_{p}^{\mathrm{T}} \mathcal{F}_{p} \xi_{p}+\mathbf{B}_{p} \boldsymbol{u}, \quad \xi_{p}(0)=\mathbf{p}_{0}^{* \mathrm{~T}} \boldsymbol{x}_{0}, \\
\xi_{q} & =\mathbf{q}_{0}^{* \mathrm{~T}} \mathbf{E}_{1}^{-1} \xi_{p}^{\mathrm{T}} \mathcal{F}_{p} \xi_{p}+\mathbf{B}_{q} \boldsymbol{u}, \\
\boldsymbol{y} & =\mathbf{C}_{p} \xi_{p}+\mathbf{C}_{q} \xi_{q}+\mathbf{D} \boldsymbol{u},
\end{aligned}
$$

where $\mathbf{A}_{p}=\mathbf{p}_{0}^{* \mathrm{~T}} \mathbf{E}_{1}^{-1} \mathbf{A}_{0} \mathbf{p}_{0} \in \mathbb{R}^{n_{p} \times n_{p}}, \mathcal{F}_{p}=\mathbf{p}_{0}^{\mathrm{T}} \mathcal{F}_{P} \mathbf{p}_{0} \in \mathbb{R}^{n_{p} \times n_{p} \times n}, \mathbf{B}_{p}=\mathbf{p}_{0}^{* \mathrm{~T}} \mathbf{E}_{1}^{-1} \mathbf{B} \in$ $\mathbb{R}^{n_{p} \times m}, \mathbf{B}_{q}=\mathbf{q}_{0}^{* \mathrm{~T}} \mathbf{E}_{1}^{-1} \mathbf{B} \in \mathbb{R}^{n_{q} \times m}, \mathbf{C}_{p}=\mathbf{C p}_{0} \in \mathbb{R}^{\ell \times n_{p}}, \mathbf{C}_{q}=\mathbf{C} \mathbf{q}_{0} \in \mathbb{R}^{\ell \times n_{q}}$. Using the transformation (5), we can rewrite the nonlinear terms of (25) as

$$
\mathbf{p}_{0}^{* \mathrm{~T}} \mathbf{E}_{1}^{-1} \xi_{p}^{\mathrm{T}} \mathcal{F}_{p} \xi_{p}=\xi_{p}^{\mathrm{T}} \mathbf{f}_{p} \xi_{p} \quad \text { and } \quad \mathbf{q}_{0}^{* \mathrm{~T}} \mathbf{E}_{1}^{-1} \xi_{p}^{\mathrm{T}} \mathcal{F}_{p} \xi_{p}=\xi_{p}^{\mathrm{T}} \mathbf{f}_{q p} \xi_{p},
$$

where $\mathbf{f}_{p} \in \mathbb{R}^{n_{p} \times n_{p} \times n_{p}}, \mathbf{f}_{q p} \in \mathbb{R}^{n_{p} \times n_{p} \times n_{q}}$. Thus, system (25) can be rewritten as

$$
\begin{aligned}
\xi_{p}^{\prime} & =\mathbf{A}_{p} \xi_{p}+\xi_{p}^{\mathrm{T}} \mathbf{f}_{p} \xi_{p}+\mathbf{B}_{p} \boldsymbol{u}, \quad \xi_{p}(0)=\mathbf{p}_{0}^{* \mathrm{~T}} \boldsymbol{x}_{0} \\
\xi_{q} & =\xi_{p}^{\mathrm{T}} \mathbf{f}_{q p} \xi_{p}+\mathbf{B}_{q} \boldsymbol{u} \\
\boldsymbol{y} & =\mathbf{C}_{p} \xi_{p}+\mathbf{C}_{q} \xi_{q}+\mathbf{D} \boldsymbol{u}
\end{aligned}
$$

From (24), we obtain

(ii) Coupling from algebraic to differential part

$$
\begin{aligned}
\xi_{q} & =\mathbf{q}_{0}^{* \mathrm{~T}} \mathbf{E}_{1}^{-1} \xi_{q}^{\mathrm{T}} \mathcal{F}_{q} \xi_{q}+\mathbf{B}_{q} \boldsymbol{u}, \\
\xi_{p}^{\prime} & =\mathbf{A}_{p} \xi_{p}+\mathbf{p}_{0}^{* \mathrm{~T}} \mathbf{E}_{1}^{-1} \xi_{q}^{\mathrm{T}} \mathcal{F}_{q} \xi_{q}+\mathbf{B}_{p} \boldsymbol{u}, \quad \xi_{p}(0)=\mathbf{p}_{0}^{* \mathrm{~T}} \boldsymbol{x}_{0}, \\
\boldsymbol{y} & =\mathbf{C}_{q} \xi_{q}+\mathbf{C}_{p} \xi_{p}+\mathbf{D} \boldsymbol{u},
\end{aligned}
$$

where $\mathcal{F}_{q}=\mathbf{q}_{0}^{\mathrm{T}} \mathcal{F}_{Q} \mathbf{q}_{0} \in \mathbb{R}^{n_{q} \times n_{q} \times n}, \mathbf{B}_{q}=\mathbf{q}_{0}^{* \mathrm{~T}} \mathbf{E}_{1}^{-1} \mathbf{B} \in \mathbb{R}^{n_{q} \times m}, \mathbf{A}_{p}=\mathbf{p}_{0}^{* \mathrm{~T}} \mathbf{E}_{1}^{-1} \mathbf{A}_{0} \mathbf{p}_{0} \in$ $\mathbb{R}^{n_{p} \times n_{p}}, \mathbf{B}_{p}=\mathbf{p}_{0}^{* \mathrm{~T}} \mathbf{E}_{1}^{-1} \mathbf{B} \in \mathbb{R}^{n_{p} \times m}$. Using the transformation (5), we can rewrite the nonlinear terms of (27) as

$$
\mathbf{q}_{0}^{* \mathrm{~T}} \mathbf{E}_{1}^{-1} \xi_{q}^{\mathrm{T}} \mathcal{F}_{q} \xi_{q}=\xi_{q}^{\mathrm{T}} \mathbf{f}_{q} \xi_{q} \quad \text { and } \quad \mathbf{p}_{0}^{* \mathrm{~T}} \mathbf{E}_{1}^{-1} \xi_{q}^{\mathrm{T}} \mathcal{F}_{q} \xi_{q}=\xi_{q}^{\mathrm{T}} \mathbf{f}_{p q} \xi_{q}
$$

where $\mathbf{f}_{q} \in \mathbb{R}^{n_{q} \times n_{q} \times n_{q}}, \mathbf{f}_{p q} \in \mathbb{R}^{n_{q} \times n_{q} \times n_{p}}$. Thus system (27) can be rewritten as

$$
\begin{aligned}
\xi_{q} & =\xi_{q}^{\mathrm{T}} \mathbf{f}_{q} \xi_{q}+\mathbf{B}_{q} \boldsymbol{u}, \\
\xi_{p}^{\prime} & =\mathbf{A}_{p} \xi_{p}+\xi_{q}^{\mathrm{T}} \mathbf{f}_{p q} \xi_{q}+\mathbf{B}_{p} \boldsymbol{u}, \quad \xi_{p}(0)=\mathbf{p}_{0}^{* \mathrm{~T}} \boldsymbol{x}_{0}, \\
\boldsymbol{y} & =\mathbf{C}_{q} \xi_{q}+\mathbf{C}_{p} \xi_{p}+\mathbf{D} \boldsymbol{u} .
\end{aligned}
$$


We see that both of the above decoupled systems (26) and (28) involve the inverse of $\mathbf{E}_{1}$, which might be computationally very expensive for large-scale applications. In the next section we discuss decoupling avoiding matrix inversion.

\section{Implicit-decoupling of index-1 quadratic DAEs}

In this section, we derive decoupled systems by using (19) instead of (20).

\subsection{Coupling from differential to algebraic parts}

Here, we also assume that $\mathcal{F}_{Q P}=\mathbf{0}, \mathcal{F}_{P Q}=\mathbf{0}, \mathcal{F}_{Q}=\mathbf{0}$, thus (19) simplifies to

$$
\mathbf{E}_{1}\left[\mathbf{P}_{0} \boldsymbol{x}^{\prime}+\mathbf{Q}_{0} \boldsymbol{x}\right]=\mathbf{A}_{0} \mathbf{P}_{0} \boldsymbol{x}+\boldsymbol{x}^{\mathrm{T}} \mathcal{F}_{P} \boldsymbol{x}+\mathbf{B} \boldsymbol{u} .
$$

Substituting $\boldsymbol{x}=\left(\begin{array}{ll}\mathbf{p}_{0} & \mathbf{q}_{0}\end{array}\right)\left(\begin{array}{ll}\xi_{p}^{\mathrm{T}} & \xi_{q}^{\mathrm{T}}\end{array}\right)^{\mathrm{T}}$ into (29), we obtain

$$
\left(\begin{array}{ll}
\mathbf{E}_{1} \mathbf{p}_{0} & \mathbf{0}
\end{array}\right)\left(\begin{array}{l}
\xi_{p} \\
\xi_{q}
\end{array}\right)^{\prime}=\left(\begin{array}{ll}
\mathbf{A}_{0} \mathbf{p}_{0} & -\mathbf{E}_{1} \mathbf{q}_{0}
\end{array}\right)\left(\begin{array}{l}
\xi_{p} \\
\xi_{q}
\end{array}\right)+\left(\begin{array}{ll}
\xi_{p}^{\mathrm{T}} & \xi_{q}^{\mathrm{T}}
\end{array}\right)\left(\begin{array}{l}
\mathbf{p}_{0}^{\mathrm{T}} \\
\mathbf{q}_{0}^{\mathrm{T}}
\end{array}\right) \mathcal{F}_{P}\left(\begin{array}{ll}
\mathbf{p}_{0} & \mathbf{q}_{0}
\end{array}\right)\left(\begin{array}{l}
\xi_{p} \\
\xi_{q}
\end{array}\right)+\mathbf{B} \boldsymbol{u} .
$$

Consider another set of matrices $\hat{\mathbf{p}}_{0} \in \mathbb{R}^{n \times n_{p}}$ and $\hat{\mathbf{q}}_{0} \in \mathbb{R}^{n \times n_{q}}$, whose columns are linearly independent, respectively. Then left multiplying (30) by $\left(\begin{array}{ll}\hat{\mathbf{p}}_{0} & \hat{\mathbf{q}}_{0}\end{array}\right)^{\mathrm{T}} \in \mathbb{R}^{n \times n}$, we obtain

$$
\left(\begin{array}{cc}
\hat{\mathbf{p}}_{0}^{\mathrm{T}} \mathbf{E}_{1} \mathbf{p}_{0} & \mathbf{0} \\
\hat{\mathbf{q}}_{0}^{\mathrm{T}} \mathbf{E}_{1} \mathbf{p}_{0} & \mathbf{0}
\end{array}\right)\left(\begin{array}{c}
\xi_{p} \\
\xi_{q}
\end{array}\right)^{\prime}=\left(\begin{array}{cc}
\hat{\mathbf{p}}_{0}^{\mathrm{T}} \mathbf{A}_{0} \mathbf{p}_{0} & -\hat{\mathbf{p}}_{0}^{\mathrm{T}} \mathbf{E}_{1} \mathbf{q}_{0} \\
\hat{\mathbf{q}}_{0}^{\mathrm{T}} \mathbf{A}_{0} \mathbf{p}_{0} & -\hat{\mathbf{q}}_{0}^{\mathrm{T}} \mathbf{E}_{1} \mathbf{q}_{0}
\end{array}\right)\left(\begin{array}{c}
\xi_{p} \\
\xi_{q}
\end{array}\right)+\left(\begin{array}{c}
\hat{\mathbf{p}}_{0}^{\mathrm{T}} \xi_{p}^{\mathrm{T}} \mathbf{p}_{0}^{\mathrm{T}} \mathcal{F} \mathbf{p}_{0} \xi_{p} \\
\hat{\mathbf{q}}_{0}^{\mathrm{T}} \xi_{p}^{\mathrm{T}} \mathbf{p}_{0}^{\mathrm{T}} \mathcal{F} \mathbf{p}_{0} \xi_{p}
\end{array}\right)+\left(\begin{array}{c}
\hat{\mathbf{p}}_{0}^{\mathrm{T}} \mathbf{B} \\
\hat{\mathbf{q}}_{0}^{\mathrm{T}} \mathbf{B}
\end{array}\right) \boldsymbol{u},
$$

since $\mathbf{p}_{0}^{\mathrm{T}} \mathcal{F}_{P} \mathbf{q}_{0}=\mathbf{0}, \mathbf{q}_{0}^{\mathrm{T}} \mathcal{F}_{P} \mathbf{p}_{0}=\mathbf{0}, \mathbf{q}_{0}^{\mathrm{T}} \mathcal{F}_{P} \mathbf{q}_{0}=\mathbf{0}$ and $\mathbf{p}_{0}^{\mathrm{T}} \mathcal{F}_{P} \mathbf{p}_{0}=\mathbf{p}_{0}^{\mathrm{T}} \mathcal{F} \mathbf{p}_{0}$. Note that if $\hat{\mathbf{q}}_{0}^{\mathrm{T}} \mathbf{E}_{1} \mathbf{p}_{0}=\mathbf{0}$ and $\hat{\mathbf{p}}_{0}^{\mathrm{T}} \mathbf{E}_{1} \mathbf{q}_{0}=\mathbf{0}$, we obtain a one-way coupled system from (31). This is archived by constructing $\hat{\mathbf{p}}_{0}$ and $\hat{\mathbf{q}}_{0}$ such that their linearly independent columns span the null spaces of $\mathbf{q}_{0}^{\mathrm{T}} \mathbf{A}_{0}^{\mathrm{T}}$ and $\mathbf{p}_{0}^{\mathrm{T}} \mathbf{E}_{0}^{\mathrm{T}}$, respectively. Let $\mathcal{F}_{p}=\mathbf{p}_{0}^{\mathrm{T}} \mathcal{F}_{\mathbf{p}_{0}} \in \mathbb{R}^{n_{p} \times n_{p} \times n}$, then (31) simplifies to

$$
\left(\begin{array}{cc}
\hat{\mathbf{p}}_{0}^{\mathrm{T}} \mathbf{E}_{1} \mathbf{p}_{0} & \mathbf{0} \\
\mathbf{0} & \mathbf{0}
\end{array}\right)\left(\begin{array}{c}
\xi_{p} \\
\xi_{q}
\end{array}\right)^{\prime}=\left(\begin{array}{cc}
\hat{\mathbf{p}}_{0}^{\mathrm{T}} \mathbf{A}_{0} \mathbf{p}_{0} & \mathbf{0} \\
\hat{\mathbf{q}}_{0}^{\mathrm{T}} \mathbf{A}_{0} \mathbf{p}_{0} & -\hat{\mathbf{q}}_{0}^{\mathrm{T}} \mathbf{E}_{1} \mathbf{q}_{0}
\end{array}\right)\left(\begin{array}{c}
\xi_{p} \\
\xi_{q}
\end{array}\right)+\left(\begin{array}{c}
\hat{\mathbf{p}}_{0}^{\mathrm{T}} \xi_{p}^{\mathrm{T}} \mathcal{F}_{p} \xi_{p} \\
\hat{\mathbf{q}}_{0}^{\mathrm{T}} \xi_{p}^{\mathrm{T}} \mathcal{F}_{p} \xi_{p}
\end{array}\right)+\left(\begin{array}{c}
\hat{\mathbf{p}}_{0}^{\mathrm{T}} \mathbf{B} \\
\hat{\mathbf{q}}_{0}^{\mathrm{T}} \mathbf{B}
\end{array}\right) \boldsymbol{u} .
$$

Using (5), we get the transformation $\hat{\mathbf{p}}_{0}^{\mathrm{T}} \xi_{p}^{\mathrm{T}} \mathcal{F}_{p} \xi_{p}=\xi_{p}^{\mathrm{T}} \mathbf{f}_{p} \xi_{p}, \hat{\mathbf{q}}_{0}^{\mathrm{T}} \xi_{p}^{\mathrm{T}} \mathcal{F}_{p} \xi_{p}=\xi_{p}^{\mathrm{T}} \mathbf{f}_{q p} \xi_{p}$, where $\mathbf{f}_{p} \in \mathbb{R}^{n_{p} \times n_{p} \times n_{p}}, \mathbf{f}_{q p} \in \mathbb{R}^{n_{p} \times n_{p} \times n_{q}}$. Using the fact that $\mathbf{E}_{1}=\mathbf{E}_{0}-\mathbf{A}_{0} \mathbf{Q}_{0}$, (32) simplifies to an implicitly decoupled system equivalent to (1) given by

$$
\begin{aligned}
\mathbf{E}_{p} \xi_{p}^{\prime} & =\mathbf{A}_{p} \xi_{p}+\xi_{p}^{\mathrm{T}} \mathbf{f}_{p} \xi_{p}+\mathbf{B}_{p} \boldsymbol{u}, \quad \xi_{p}(0)=\mathbf{p}_{0}^{* \mathrm{~T}} \boldsymbol{x}_{0}, \\
\mathbf{E}_{q} \xi_{q} & =\mathbf{A}_{q} \xi_{p}+\xi_{p}^{\mathrm{T}} \mathbf{f}_{q p} \xi_{p}+\mathbf{B}_{q} \boldsymbol{u}, \\
\boldsymbol{y} & =\mathbf{C}_{p} \xi_{p}+\mathbf{C}_{q} \xi_{q}+\mathbf{D} \boldsymbol{u},
\end{aligned}
$$

where $\mathbf{E}_{p}=\hat{\mathbf{p}}_{0}^{\mathrm{T}} \mathbf{E}_{0} \mathbf{p}_{0}, \mathbf{A}_{p}=\hat{\mathbf{p}}_{0}^{\mathrm{T}} \mathbf{A}_{0} \mathbf{p}_{0} \in \mathbb{R}^{n_{p} \times n_{p}}, \mathbf{B}_{p}=\hat{\mathbf{p}}_{0}^{\mathrm{T}} \mathbf{B} \in \mathbb{R}^{n_{p} \times m}, \mathbf{E}_{q}=-\hat{\mathbf{q}}_{0}^{\mathrm{T}} \mathbf{A}_{0} \mathbf{q}_{0} \in$ $\mathbb{R}^{n_{q} \times n_{q}}, \mathbf{A}_{q}=\hat{\mathbf{q}}_{0}^{\mathrm{T}} \mathbf{A}_{0} \mathbf{p}_{0} \in \mathbb{R}^{n_{q} \times n_{p}}, \mathbf{B}_{q}=\hat{\mathbf{q}}_{0}^{\mathrm{T}} \mathbf{B} \in \mathbb{R}^{n_{q} \times m}, \mathbf{C}_{p}=\mathbf{C p}_{0} \in \mathbb{R}^{\ell \times n_{p}}, \mathbf{C}_{q}=\mathbf{C q}_{0} \in$ $\mathbb{R}^{\ell \times n_{q}}$. 


\subsection{Coupling from algebraic to differential part}

To obtain this coupling, we assume that $\mathcal{F}_{Q P}=\mathbf{0}, \mathcal{F}_{P Q}=\mathbf{0}, \mathcal{F}_{P}=\mathbf{0}$, thus (19) simplifies to

$$
\mathbf{E}_{1}\left[\mathbf{P}_{0} \boldsymbol{x}^{\prime}+\mathbf{Q}_{0} \boldsymbol{x}\right]=\mathbf{A}_{0} \mathbf{P}_{0} \boldsymbol{x}+\boldsymbol{x}^{\mathrm{T}} \mathcal{F}_{Q} \boldsymbol{x}+\mathbf{B} \boldsymbol{u} .
$$

Substituting $\boldsymbol{x}=\left(\begin{array}{ll}\mathbf{q}_{0} & \mathbf{p}_{0}\end{array}\right)\left(\begin{array}{ll}\xi_{q}^{\mathrm{T}} & \xi_{p}^{\mathrm{T}}\end{array}\right)^{\mathrm{T}}$ into (34), we obtain

$$
\left(\begin{array}{ll}
\mathbf{0} & \mathbf{E}_{1} \mathbf{p}_{0}
\end{array}\right)\left(\begin{array}{l}
\xi_{q} \\
\xi_{p}
\end{array}\right)^{\prime}=\left(\begin{array}{ll}
-\mathbf{E}_{1} \mathbf{q}_{0} & \mathbf{A}_{0} \mathbf{p}_{0}
\end{array}\right)\left(\begin{array}{l}
\xi_{q} \\
\xi_{p}
\end{array}\right)+\left(\begin{array}{ll}
\xi_{q}^{\mathrm{T}} & \xi_{p}^{\mathrm{T}}
\end{array}\right)\left(\begin{array}{l}
\mathbf{q}_{0}^{\mathrm{T}} \\
\mathbf{p}_{0}^{\mathrm{T}}
\end{array}\right) \mathcal{F}_{Q}\left(\begin{array}{ll}
\mathbf{q}_{0} & \mathbf{p}_{0}
\end{array}\right)\left(\begin{array}{l}
\xi_{q} \\
\xi_{p}
\end{array}\right)+\mathbf{B} \boldsymbol{u} .
$$

Consider another set of matrices $\hat{\mathbf{p}}_{0} \in \mathbb{R}^{n \times n_{p}}$ and $\hat{\mathbf{q}}_{0} \in \mathbb{R}^{n \times n_{q}}$, whose columns are linearly independent, respectively. Left multiplying (35) by $\left(\begin{array}{ll}\hat{\mathbf{q}}_{0} & \hat{\mathbf{p}}_{0}\end{array}\right)^{\mathrm{T}} \in \mathbb{R}^{n \times n}$, we obtain:

$$
\left(\begin{array}{cc}
\mathbf{0} & \hat{\mathbf{q}}_{0}^{\mathrm{T}} \mathbf{E}_{1} \mathbf{p}_{0} \\
\mathbf{0} & \hat{\mathbf{p}}_{0}^{\mathrm{T}} \mathbf{E}_{1} \mathbf{p}_{0}
\end{array}\right)\left(\begin{array}{c}
\xi_{q} \\
\xi_{p}
\end{array}\right)^{\prime}=\left(\begin{array}{cc}
-\hat{\mathbf{q}}_{0}^{\mathrm{T}} \mathbf{E}_{1} \mathbf{q}_{0} & \hat{\mathbf{q}}_{0}^{\mathrm{T}} \mathbf{A}_{0} \mathbf{p}_{0} \\
-\hat{\mathbf{p}}_{0}^{\mathrm{T}} \mathbf{E}_{1} \mathbf{q}_{0} & \hat{\mathbf{p}}_{0}^{\mathrm{T}} \mathbf{A}_{0} \mathbf{p}_{0}
\end{array}\right)\left(\begin{array}{c}
\xi_{q} \\
\xi_{p}
\end{array}\right)+\left(\begin{array}{c}
\hat{\mathbf{q}}_{0}^{\mathrm{T}} \xi_{q}^{\mathrm{T}} \mathbf{q}_{0}^{\mathrm{T}} \mathcal{F} \mathbf{q}_{0} \xi_{q} \\
\hat{\mathbf{p}}_{0}^{\mathrm{T}} \xi_{q}^{\mathrm{T}} \mathbf{q}_{0}^{\mathrm{T}} \mathcal{F} \mathbf{q}_{0} \xi_{q}
\end{array}\right)+\left(\begin{array}{c}
\hat{\mathbf{q}}_{0}^{\mathrm{T}} \mathbf{B} \\
\hat{\mathbf{p}}_{0}^{\mathrm{T}} \mathbf{B}
\end{array}\right) \boldsymbol{u}
$$

Analogous to (31), if $\hat{\mathbf{q}}_{0}^{\mathrm{T}} \mathbf{A}_{0} \mathbf{p}_{0}=\mathbf{0}$ and $\hat{\mathbf{p}}_{0}^{\mathrm{T}} \mathbf{E}_{1} \mathbf{q}_{0}=-\hat{\mathbf{p}}_{0}^{\mathrm{T}} \mathbf{A}_{0} \mathbf{q}_{0}=\mathbf{0}$, we can obtain a oneway coupled system from (36). Similarly, we construct $\hat{\mathbf{p}}_{0}$ and $\hat{\mathbf{q}}_{0}$ such that their linearly independent columns span the null spaces of $\mathbf{q}_{0}^{\mathrm{T}} \mathbf{A}_{0}^{\mathrm{T}}$ and $\mathbf{p}_{0}^{\mathrm{T}} \mathbf{A}_{0}^{\mathrm{T}}$, respectively. Let $\mathcal{F}_{q}=$ $\mathbf{q}_{0}^{\mathrm{T}} \mathcal{F} \mathbf{q}_{0} \in \mathbb{R}^{n_{q} \times n_{q} \times n}$, then (36) can be written as

$$
\left(\begin{array}{cc}
\mathbf{0} & \mathbf{0} \\
\mathbf{0} & \hat{\mathbf{p}}_{0}^{\mathrm{T}} \mathbf{E}_{1} \mathbf{p}_{0}
\end{array}\right)\left(\begin{array}{c}
\xi_{q} \\
\xi_{p}
\end{array}\right)^{\prime}=\left(\begin{array}{cc}
-\hat{\mathbf{q}}_{0}^{\mathrm{T}} \mathbf{E}_{1} \mathbf{q}_{0} & \mathbf{0} \\
\mathbf{0} & \hat{\mathbf{p}}_{0}^{\mathrm{T}} \mathbf{A}_{0} \mathbf{p}_{0}
\end{array}\right)\left(\begin{array}{l}
\xi_{q} \\
\xi_{p}
\end{array}\right)+\left(\begin{array}{c}
\hat{\mathbf{q}}_{0}^{\mathrm{T}} \xi_{q}^{\mathrm{T}} \mathcal{F}_{q} \xi_{q} \\
\hat{\mathbf{p}}_{0}^{\mathrm{T}} \xi_{q}^{\mathrm{T}} \mathcal{F}_{q} \xi_{q}
\end{array}\right)+\left(\begin{array}{c}
\hat{\mathbf{q}}_{0}^{\mathrm{T}} \mathbf{B} \\
\hat{\mathbf{p}}_{0}^{\mathrm{T}} \mathbf{B}
\end{array}\right) \boldsymbol{u} .
$$

Using (5), we get the transformation $\hat{\mathbf{q}}_{0}^{\mathrm{T}} \xi_{q}^{\mathrm{T}} \mathcal{F}_{q} \xi_{q}=\xi_{q}^{\mathrm{T}} \mathbf{f}_{q} \xi_{q}, \hat{\mathbf{p}}_{0}^{\mathrm{T}} \xi_{q}^{\mathrm{T}} \mathcal{F}_{q} \xi_{q}=\xi_{q}^{\mathrm{T}} \mathbf{f}_{p q} \xi_{q}$, where

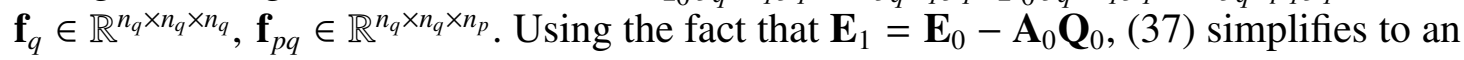
implicit decoupled system equivalent to (1) given by

$$
\begin{aligned}
\mathbf{E}_{q} \xi_{q} & =\xi_{q}^{\mathrm{T}} \mathbf{f}_{q} \xi_{q}+\mathbf{B}_{q} \boldsymbol{u}, \\
\mathbf{E}_{p} \xi_{p}^{\prime} & =\mathbf{A}_{p} \xi_{p}+\xi_{q}^{\mathrm{T}} \mathbf{f}_{p q} \xi_{q}+\mathbf{B}_{p} \boldsymbol{u}, \quad \xi_{p}(0)=\mathbf{p}_{0}^{* \mathrm{~T}} \boldsymbol{x}_{0}, \\
\boldsymbol{y} & =\mathbf{C}_{p} \xi_{p}+\mathbf{C}_{q} \xi_{q}+\mathbf{D} \boldsymbol{u},
\end{aligned}
$$

where $\mathbf{E}_{p}=\hat{\mathbf{p}}_{0}^{\mathrm{T}} \mathbf{E}_{0} \mathbf{p}_{0}, \mathbf{A}_{p}=\hat{\mathbf{p}}_{0}^{\mathrm{T}} \mathbf{A}_{0} \mathbf{p}_{0} \in \mathbb{R}^{n_{p} \times n_{p}}, \mathbf{B}_{p}=\hat{\mathbf{p}}_{0}^{\mathrm{T}} \mathbf{B} \in \mathbb{R}^{n_{p} \times m}, \mathbf{E}_{q}=-\hat{\mathbf{q}}_{0}^{\mathrm{T}} \mathbf{A}_{0} \mathbf{q}_{0} \in$ $\mathbb{R}^{n_{q} \times n_{q}}, \mathbf{B}_{q}=\hat{\mathbf{q}}_{0}^{\mathrm{T}} \mathbf{B} \in \mathbb{R}^{n_{q} \times m}, \mathbf{C}_{p}=\mathbf{C} \mathbf{p}_{0} \in \mathbb{R}^{\ell \times n_{p}}, \mathbf{C}_{q}=\mathbf{C q} \mathbf{q}_{0} \in \mathbb{R}^{\ell \times n_{q}}$.

Due to their equivalence, system (38) is solvable if and only if (1) is solvable. We observe that,since $\mathbf{E}_{q}$ is nonsingular, (38a) can be re-written into a classical fixed point equation which can, e.g., be solved using Newton's method. After solving (38a), $\xi_{q}^{\mathrm{T}} \mathbf{f}_{p q} \xi_{q}$ can be considered an extra input for the differential subsystem (38b) which can be solved using any existing numerical integration technique, such as implicit Euler method. Finally, the output solution is obtained through (38c). 


\section{Index-aware pMOR (IpMOR) method for quadratic parameterized DAEs}

After decoupling system (1) into a one-way coupled system, we are ready to apply model order reduction to the one-way coupled system rather than to (1). Here, we propose an IpMOR method for quadratic parametrized DAEs. The IpMOR method is an extension of the IMOR method for DAEs proposed in [3,9]. We consider the weakly coupled systems (33) and (38) discussed in Section 4 to construct the corresponding IpROMs for weakly coupled systems from the differential to algebraic part, and from the algebraic to differential part, respectively. In order to obtain an IpROM for the system coupled from the differential to the algebraic part, we consider system (33) in parametrized form

$$
\begin{aligned}
\mathbf{E}_{p}(\mu) \xi_{p}^{\prime} & =\mathbf{A}_{p}(\mu) \xi_{p}+\xi_{p}^{\mathrm{T}} \mathbf{f}_{p}(\mu) \xi_{p}+\mathbf{B}_{p}(\mu) \boldsymbol{u}, \quad \xi_{p}(0)=\mathbf{p}_{0}^{* \mathrm{~T}} \boldsymbol{x}_{0} \\
\mathbf{E}_{q}(\mu) \xi_{q} & =\mathbf{A}_{q}(\mu) \xi_{p}+\xi_{p}^{\mathrm{T}} \mathbf{f}_{q p}(\mu) \xi_{p}+\mathbf{B}_{q}(\mu) \boldsymbol{u} \\
\boldsymbol{y} & =\mathbf{C}_{p} \xi_{p}(\mu)+\mathbf{C}_{q}(\mu) \xi_{q}+\mathbf{D}(\mu) \boldsymbol{u}
\end{aligned}
$$

We construct projection matrices $\mathbf{V}_{p} \in \mathbb{R}^{n_{p} \times r_{1}}, r_{1} \ll n_{p}$ and $\mathbf{V}_{q} \in \mathbb{R}^{n_{q} \times r_{2}}, r_{2} \ll n_{q}$ for the differential and algebraic parts, respectively, using the existing pMOR based on Galerkin projection. The reduced-order system is as below,

$$
\begin{aligned}
\mathbf{E}_{p_{r}}(\mu) \xi_{p_{r}}^{\prime} & =\mathbf{A}_{p_{r}}(\mu) \xi_{p_{r}}+\xi_{p_{r}}^{\mathrm{T}} \hat{\mathbf{f}}_{p_{r}}(\mu) \xi_{p_{r}}+\mathbf{B}_{p_{r}}(\mu) \boldsymbol{u}, \quad \xi_{p_{r}}(0)=\mathbf{V}_{p}^{\mathrm{T}} \xi_{p}(0), \\
\mathbf{E}_{q_{r}}(\mu) \xi_{q_{r}} & =\mathbf{A}_{q_{r}}(\mu) \xi_{q_{r}}+\xi_{p_{r}}^{\mathrm{T}} \hat{\mathbf{f}}_{p_{r}}(\mu) \xi_{p_{r}}+\mathbf{B}_{q_{r}}(\mu) \boldsymbol{u}, \\
\boldsymbol{y}_{r} & =\mathbf{C}_{p_{r}} \xi_{p_{r}}(\mu)+\mathbf{C}_{q_{r}}(\mu) \xi_{q_{r}}+\mathbf{D}(\mu) \boldsymbol{u}
\end{aligned}
$$

where $\mathbf{E}_{p_{r}}(\mu)=\mathbf{V}_{p}^{\mathrm{T}} \mathbf{E}_{p}(\mu) \mathbf{V}_{p}, \mathbf{A}_{p_{r}}(\mu)=\mathbf{V}_{p}^{\mathrm{T}} \mathbf{A}_{p}(\mu) \mathbf{V}_{p} \in \mathbb{R}^{r_{1} \times r_{1}}, \mathbf{B}_{p_{r}}(\mu)=\mathbf{V}_{p}^{\mathrm{T}} \mathbf{B}_{p}(\mu) \in \mathbb{R}^{r_{1} \times m}$, $\mathbf{E}_{q_{r}}(\mu)=\mathbf{V}_{q}^{\mathrm{T}} \mathbf{E}_{q}(\mu) \mathbf{V}_{q} \in \mathbb{R}^{r_{2} \times r_{2}}, \mathbf{A}_{q_{r}}(\mu)=\mathbf{V}_{q}^{\mathrm{T}} \mathbf{A}_{q}(\mu) \mathbf{V}_{q} \in \mathbb{R}^{r_{1} \times r_{2}}, \mathbf{B}_{q_{r}}(\mu)=\mathbf{V}_{q}^{\mathrm{T}} \mathbf{B}_{q}(\mu) \in \mathbb{R}^{r_{2} \times m}$ and $\mathbf{C}_{p_{r}}(\mu)=\mathbf{C}_{p}(\mu) \mathbf{V}_{p} \in \mathbb{R}^{r_{1} \times \ell}, \mathbf{C}_{q_{r}}(\mu)=\mathbf{C}_{q}(\mu) \mathbf{V}_{q} \in \mathbb{R}^{r_{2} \times \ell}$. The nonlinear terms are obtained using the transformation (5) leading to: $\mathbf{V}_{p}^{\mathrm{T}} \xi_{p_{r}}^{\mathrm{T}} \mathbf{f}_{p_{r}}(\mu) \xi_{p_{r}}=\xi_{p_{r}}^{\mathrm{T}} \hat{\mathbf{f}}_{p_{r}}(\mu) \xi_{p_{r}}$ where $\mathbf{f}_{p_{r}}(\mu)=\mathbf{V}_{p}^{\mathrm{T}} \mathbf{f}_{p}(\mu) \mathbf{V}_{p} \in \mathbb{R}^{r_{1} \times r_{1} \times n_{p}}, \hat{\mathbf{f}}_{p_{r}} \in \mathbb{R}^{r_{1} \times r_{1} \times r_{1}}$ and $\mathbf{V}_{q}^{\mathrm{T}} \xi_{p_{r}}^{\mathrm{T}} \mathbf{f}_{q p_{r}}(\mu) \xi_{p_{r}}=\xi_{p_{r}}^{\mathrm{T}} \hat{\mathbf{f}}_{q p_{r}}(\mu) \xi_{p_{r}}$ where $\mathbf{f}_{q p_{r}}(\mu)=\mathbf{V}_{p}^{\mathrm{T}} \mathbf{f}_{q p}(\mu) \mathbf{V}_{p} \in \mathbb{R}^{r_{1} \times r_{1} \times n_{q}}, \hat{\mathbf{f}}_{q p_{r}} \in \mathbb{R}^{r_{1} \times r_{1} \times r_{2}}$.

Next, we obtain an IpROM for the system (38) coupled from the algebraic to the differential part,

$$
\begin{aligned}
\mathbf{E}_{q}(\mu) \xi_{q} & =\xi_{q}^{\mathrm{T}} \mathbf{f}_{q}(\mu) \xi_{q}+\mathbf{B}_{q}(\mu) \boldsymbol{u}, \\
\mathbf{E}_{p}(\mu) \xi_{p}^{\prime} & =\mathbf{A}_{p}(\mu) \xi_{p}+\xi_{q}^{\mathrm{T}} \mathbf{f}_{p q}(\mu) \xi_{q}+\mathbf{B}_{p}(\mu) \boldsymbol{u}, \quad \xi_{p}(0)=\mathbf{p}_{0}^{* \mathrm{~T}} \boldsymbol{x}_{0}, \\
\boldsymbol{y} & =\mathbf{C}_{p}(\mu) \xi_{p}+\mathbf{C}_{q}(\mu) \xi_{q}+\mathbf{D}(\mu) \boldsymbol{u} .
\end{aligned}
$$

Likewise, the projection matrices $\mathbf{V}_{q} \in \mathbb{R}^{n_{q} \times r_{1}}, r_{1} \ll n_{q}$, and $\mathbf{V}_{p} \in \mathbb{R}^{n_{p} \times r_{2}}, r_{2} \ll n_{p}$ for the algebraic and differential parts, are constructed separately, using the existing pMOR 
methods. The resulting reduced-order system is

$$
\begin{aligned}
\mathbf{E}_{q_{r}}(\mu) \xi_{q_{r}} & =\xi_{q_{r}}^{\mathrm{T}} \hat{\mathbf{f}}_{q_{r}}(\mu) \xi_{q_{r}}+\mathbf{B}_{q_{r}}(\mu) \boldsymbol{u}, \\
\mathbf{E}_{p_{r}}(\mu) \xi_{p_{r}}^{\prime} & =\mathbf{A}_{p_{r}}(\mu) \xi_{p_{r}}+\xi_{q_{r}}^{\mathrm{T}} \hat{\mathbf{f}}_{p q_{r}}(\mu) \xi_{q_{r}}+\mathbf{B}_{p_{r}}(\mu) \boldsymbol{u}, \quad \xi_{p_{r}}(0)=\mathbf{V}_{p}^{\mathrm{T}} \xi_{p}(0), \\
\boldsymbol{y}_{r} & =\mathbf{C}_{p}(\mu) \xi_{p_{r}}+\mathbf{C}_{q_{r}}(\mu) \xi_{q_{r}}+\mathbf{D}(\mu) \boldsymbol{u},
\end{aligned}
$$

where $\mathbf{E}_{p_{r}}(\mu)=\mathbf{V}_{p}^{\mathrm{T}} \mathbf{E}_{p}(\mu) \mathbf{V}_{p}, \mathbf{A}_{p_{r}}(\mu)=\mathbf{V}_{p}^{\mathrm{T}} \mathbf{A}_{p}(\mu) \mathbf{V}_{p} \in \mathbb{R}^{r_{2} \times r_{2}}, \mathbf{B}_{p_{r}}(\mu)=\mathbf{V}_{p}^{\mathrm{T}} \mathbf{B}_{p}(\mu) \in \mathbb{R}^{r_{2} \times m}$, $\mathbf{E}_{q_{r}}(\mu)=\mathbf{V}_{q}^{\mathrm{T}} \mathbf{E}_{q}(\mu) \mathbf{V}_{q} \in \mathbb{R}^{r_{1} \times r_{1}}, \mathbf{B}_{q_{r}}(\mu)=\mathbf{V}_{q}^{\mathrm{T}} \mathbf{B}_{q}(\mu) \in \mathbb{R}^{r_{1} \times m}$ and $\mathbf{C}_{p_{r}}(\mu)=\mathbf{C}_{p}(\mu) \mathbf{V}_{p} \in$ $\mathbb{R}^{r_{2} \times \ell}, \mathbf{C}_{q_{r}}(\mu)=\mathbf{C}_{q}(\mu) \mathbf{V}_{q} \in \mathbb{R}^{r_{1} \times \ell}$. The nonlinear terms are obtained using the transformation (5) leading to: $\mathbf{V}_{q}^{\mathrm{T}} \xi_{q_{r}}^{\mathrm{T}} \mathbf{f}_{q_{r}}(\mu) \xi_{q_{r}}=\xi_{q_{r}}^{\mathrm{T}} \hat{\mathbf{f}}_{q_{r}}(\mu) \xi_{q_{r}}$, where $\mathbf{f}_{q_{r}}(\mu)=\mathbf{V}_{q}^{\mathrm{T}} \mathbf{f}_{q}(\mu) \mathbf{V}_{q} \in$ $\mathbb{R}^{r_{1} \times r_{1} \times n_{q}}, \hat{\mathbf{f}}_{q_{r}} \in \mathbb{R}^{r_{1} \times r_{1} \times r_{1}}$ and $\mathbf{V}_{p}^{\mathrm{T}} \xi_{q_{r}}^{\mathrm{T}} \mathbf{f}_{p q_{r}}(\mu) \xi_{q_{r}}=\xi_{q_{r}}^{\mathrm{T}} \hat{\mathbf{f}}_{p q_{r}}(\mu) \xi_{q_{r}}$, where $\mathbf{f}_{p q_{r}}(\mu)=\mathbf{V}_{p}^{\mathrm{T}} \mathbf{f}_{q p}(\mu) \mathbf{V}_{p} \in$ $\mathbb{R}^{r_{1} \times r_{1} \times n_{p}}, \hat{\mathbf{f}}_{p q_{r}} \in \mathbb{R}^{r_{1} \times r_{1} \times r_{2}}$.

The next step is to construct projection matrices $\mathbf{V}_{p}$ and $\mathbf{V}_{q}$ that lead to a good ROM with small approximation error $\left\|\boldsymbol{y}-\boldsymbol{y}_{r}\right\|$ in a suitable norm $\|$.$\| for every arbitrary$ input $\boldsymbol{u}(t)$ and parameter $\mu$. There are many existing pMOR methods such as the robust pMOR algorithm in [11], based on implicit multi-moment matching or snapshot methods such as the reduced basis methods [12]. See [1] for a general discussion of pMOR methods. The snapshot methods are not flexible for input dependent systems as considered in this work, since the snapshots depends on proper sampling of the inputs. As a result, the ROM may only be accurate for those training inputs which are used to generate the snapshots, and may loose accuracy for the inputs other than the training inputs. Therefore, input-independent pMOR methods are more appropriate for input-dependent systems. Here, we choose the pMOR method based on implicit multimoment-matching, because of its simplicity, low complexity, and input independency, but other pMOR approaches could also be used, such as interpolation of local bases and interpolation of local matrices, see, e.g., [1].

\section{Construction of projection matrices using the implicit moment-matching method}

In this section, we discuss how to construct the projection matrices $\mathbf{V}_{p}$ and $\mathbf{V}_{q}$ for the differential and algebraic subsystems, respectively, using the pMOR method based on implicit multi-moment matching.

\subsection{Construction of the projection matrix $\mathbf{V}_{p}$}

We discuss constructing the projection matrix $\mathbf{V}_{p}$ for either (40) or (42). We can observe that the nonlinear term $\xi_{q}^{\mathrm{T}} \mathbf{f}_{p q}(\mu) \xi_{q}$ can be treated as part of the input of the differential subsystem (41b), since it is known after first simulating the algebraic subsystem (41a). In general, we cannot ignore the nonlinear term in (39a) during the reduction but for most of our applications, $\xi_{p}^{\mathrm{T}} \mathbf{f}_{p}(\mu) \xi_{p}$ is negligible. Thus, we consider only the linear 
part of the differential subsystem in (39a) or (41b),

$$
\begin{aligned}
\mathbf{E}_{p}(\mu) \xi_{p}^{\prime} & =\mathbf{A}_{p}(\mu) \xi_{p}+\mathbf{B}_{p}(\mu) \boldsymbol{u}, \quad \xi_{p}(0)=\xi_{p_{0}}, \\
\boldsymbol{y}_{p} & =\mathbf{C}_{p}(\mu) \xi_{p} .
\end{aligned}
$$

Taking the Laplace transform of (43a) and assuming $\xi_{p}(0)=0^{1}$, we obtain

$$
\left(s \mathbf{E}_{p}(\mu)-\mathbf{A}_{p}(\mu)\right) \mathbf{X}_{p}(s)=\mathbf{B}_{p}(\mu) \mathbf{U}(s) .
$$

The state $\mathbf{X}_{p}(s)$ is the Laplace transform of the unknown vector $\xi_{p}(t)$ in (43a). Assuming each of $\mathbf{E}_{p}(\mu), \mathbf{A}_{p}(\mu), \mathbf{B}_{p}(\mu)$ exhibits an affine dependence w.r.t. the parameter $\mu$, then also (45) exhibits an affine dependence of the form

$$
\left(\tilde{\mathbf{E}}_{0}+\tilde{s}_{1} \tilde{\mathbf{E}}_{1}+\cdots+\tilde{s}_{\alpha} \tilde{\mathbf{E}}_{\alpha}\right) \mathbf{X}_{p}(s)=\left(\tilde{\mathbf{B}}_{0}+\tilde{s}_{1} \tilde{\mathbf{B}}_{1}+\cdots+\tilde{s}_{\beta} \tilde{\mathbf{B}}_{\beta}\right) \mathbf{U}(s),
$$

where $\tilde{\mathbf{E}}_{i}$ and $\tilde{\mathbf{B}}_{i}$ are the coefficients of (45) after substituting the affine expansion (2) of each of $\mathbf{E}_{p}(\mu), \mathbf{A}_{p}(\mu), \mathbf{B}_{p}(\mu)$. Here, the newly defined parameters $\tilde{s}_{i}, i=1, \ldots, k$, where $k=\max (\alpha, \beta)$, might be some functions (rational, polynomial) of the original parameters $\mu$ and the Laplace variable $s$. The state $\mathbf{X}_{p}(s)$ in (46) can be expanded into a Taylor series at an expansion point $\tilde{s}^{0}=\left(\tilde{s}_{1}^{0}, \tilde{s}_{2}^{0}, \ldots, \tilde{s}_{k}^{0}\right)$, as follows:

$$
\begin{aligned}
\mathbf{X}_{p}(s)= & {\left[\mathrm{I}-\left(\sigma_{1} \mathbf{M}_{1}+\cdots+\sigma_{\alpha} \mathbf{M}_{\alpha}\right)\right]^{-1} \tilde{\mathbf{E}}^{-1}\left[\tilde{\mathbf{B}}+\sigma_{1} \tilde{\mathbf{B}}_{1}+\cdots+\sigma_{\beta} \tilde{\mathbf{B}}_{\beta}\right] \mathbf{U}(s), } \\
= & \sum_{m=0}^{\infty}\left[\sigma_{1} \mathbf{M}_{1}+\cdots+\sigma_{\alpha} \mathbf{M}_{\alpha}\right]^{m}\left[\mathbf{B}_{M_{0}}+\sigma_{1} \mathbf{B}_{M_{1}}+\cdots+\sigma_{\beta} \mathbf{B}_{M_{\beta}}\right] \mathbf{U}(s), \\
= & {\left[\mathbf{B}_{M_{0}}+\sigma_{1} \mathbf{B}_{M_{1}}+\cdots+\sigma_{\beta} \mathbf{B}_{M_{\beta}}\right] \mathbf{U}(s) } \\
& +\left[\sigma_{1} \mathbf{M}_{1}+\cdots+\sigma_{\alpha} \mathbf{M}_{\alpha}\right]\left[\mathbf{B}_{M_{0}}+\sigma_{1} \mathbf{B}_{M_{1}}+\cdots+\sigma_{\beta} \mathbf{B}_{M_{\beta}}\right] \mathbf{U}(s)+\cdots \\
& +\left[\sigma_{1} \mathbf{M}_{1}+\cdots+\sigma_{\alpha} \mathbf{M}_{\alpha}\right]^{j}\left[\mathbf{B}_{M_{0}}+\sigma_{1} \mathbf{B}_{M_{1}}+\cdots+\sigma_{\beta} \mathbf{B}_{M_{\beta}}\right] \mathbf{U}(s)+\cdots,
\end{aligned}
$$

where $\sigma_{i}=\tilde{s}_{i}-\tilde{s}_{i}^{0}, \tilde{\mathbf{E}}=\tilde{\mathbf{E}}_{0}+\tilde{s}_{1}^{0} \tilde{\mathbf{E}}_{1}+\cdots+\tilde{s}_{\alpha}^{0} \tilde{\mathbf{E}}_{\alpha}, \mathbf{M}_{i}=-\tilde{\mathbf{E}}^{-1} \tilde{\mathbf{E}}_{i}, i=1,2, \ldots, \alpha$, and $\mathbf{B}_{M_{0}}=\tilde{\mathbf{E}}^{-1} \tilde{\mathbf{B}}, \mathbf{B}_{M_{i}}=\tilde{\mathbf{E}}^{-1} \tilde{\mathbf{B}}_{i}, i=1,2, \ldots, \beta, \tilde{\mathbf{B}}=\tilde{\mathbf{B}}_{0}+\tilde{s}_{1}^{0} \tilde{\mathbf{B}}_{1}+\cdots+\tilde{s}_{\beta}^{0} \tilde{\mathbf{B}}_{\beta}$. Using the coefficients in (47) we can define a subspace $\mathcal{R}$ spanned by the vectors in $\mathbf{R}_{j}$ given by

$$
\mathcal{R}=\operatorname{colspan}\left\{\mathbf{R}_{0}, \mathbf{R}_{1}, \ldots, \mathbf{R}_{\mathrm{j}}, \ldots, \mathbf{R}_{\mathrm{m}}\right\},
$$

\footnotetext{
${ }^{1}$ Let $\xi_{p}=\tilde{\xi}_{p}-\xi_{p_{0}}$, then system (43) can be transformed into a system with zero initial condition given by [2]

$$
\mathbf{E}_{p}(\mu) \tilde{\xi}_{p}^{\prime}=\mathbf{A}_{p}(\mu) \tilde{\xi}_{p}+\tilde{\mathbf{B}}_{p}(\mu) \tilde{\boldsymbol{u}}, \boldsymbol{y}_{p}=\mathbf{C}_{p} \tilde{\xi}_{p}(\mu)+\mathbf{C}_{p} \xi_{p_{0}}, \quad \tilde{\xi}_{p}(0)=0,
$$

where $\tilde{\mathbf{B}}_{p}(\mu)=\left[\mathbf{B}_{p}(\mu), \mathbf{A}_{p} \xi_{p_{0}}\right] \in \mathbb{R}^{n_{p} \times(m+1)}, \tilde{\boldsymbol{u}}=\left[\boldsymbol{u}^{T}, 1\right]^{T}$.
} 
where

$$
\begin{aligned}
& \mathbf{R}_{0}=\left[\mathbf{B}_{M_{0}}, \mathbf{B}_{M_{1}}, \ldots, \mathbf{B}_{M_{\beta}}\right], \mathbf{R}_{1}=\left[\mathbf{M}_{1} \mathbf{R}_{0}, \mathbf{M}_{2} \mathbf{R}_{0}, \ldots, \mathbf{M}_{\alpha} \mathbf{R}_{0}\right], \ldots, \\
& \mathbf{R}_{m}=\left[\mathbf{M}_{1} \mathbf{R}_{m-1}, \mathbf{M}_{2} \mathbf{R}_{m-1}, \ldots, \mathbf{M}_{\alpha} \mathbf{R}_{m-1}\right] .
\end{aligned}
$$

The columns of the required projection matrix $\mathbf{V}_{p}$ constitute an orthonormal basis of the subspace $\mathcal{R}$ that can be constructed by the Algorithm 1 in [11]. Note that the size of the ROM is equal to the number of columns in $\mathbf{V}_{p}$. To avoid exponential increase of the number of columns in $\mathbf{V}_{p}$, it is preferred to use only the first few $\mathbf{R}_{j}$. However, this may lead to ROMs which are not accurate for the whole frequency range. Therefore, multiple expansion points need to be used. Given a group of expansion points $\tilde{s}_{i}^{0}, i=1, \ldots, \ell$, where $i$ indicates the $i$ th expansion point, a matrix $\mathbf{V}_{\tilde{s}_{i}^{0}}$ can be computed for each $\tilde{s}_{i}^{0}$ as

$$
\operatorname{range}\left\{\mathbf{V}_{\tilde{s}_{i}^{0}}\right\}=\operatorname{colspan}\left\{\mathbf{R}_{0}, \mathbf{R}_{1}, \ldots, \mathbf{R}_{r}\right\}_{\tilde{s}_{i}^{0}} .
$$

The desired orthonormal basis matrix $\mathbf{V}_{p}$ is obtained from orthogonalization of the column vector in all matrices $\mathbf{V}_{\tilde{s}_{i}^{0}}$, i.e.,

$$
\mathbf{V}_{p}=\operatorname{orth}\left\{\mathbf{V}_{\tilde{s}_{1}^{0}}, \mathbf{V}_{\tilde{s}_{2}^{0}}, \ldots, \mathbf{V}_{\tilde{s}_{\ell}^{0}}\right\}
$$

\subsection{Construction of the projection matrix $\mathbf{V}_{q}$}

The same technique discussed in the previous subsection to construct $\mathbf{V}_{p}$ for the differential subsystem can also be used to construct the projection matrix $\mathbf{V}_{q}$ for the algebraic subsystem of either (39) or (41). Taking the algebraic part (39b) as an example, we consider also the linear part,

$$
\begin{aligned}
\mathbf{E}_{q}(\mu) \xi_{q} & =\mathbf{A}_{q}(\mu) \xi_{p}+\mathbf{B}_{q}(\mu) \boldsymbol{u}, \\
\boldsymbol{y}_{q} & =\mathbf{C}_{q}(\mu) \xi_{q}
\end{aligned}
$$

Substituting $\xi_{p}=\mathbf{V}_{p} \xi_{p_{r}}$ into (50a), where $\mathbf{V}_{p}$ is the already computed projection matrix for the differential subsystem, leads to

$$
\mathbf{E}_{q}(\mu) \tilde{\xi}_{q}=\mathbf{A}_{q}(\mu) \mathbf{V}_{p} \xi_{p_{r}}+\mathbf{B}_{q}(\mu) \boldsymbol{u} .
$$

where $\tilde{\xi}_{q}$ approximates the algebraic variable $\xi_{q}$ in (50). Here, $\xi_{p_{r}}$ can be considered as an input from the differential subsystem. Then (51) can be written as

$$
\mathbf{E}_{q}(\mu) \tilde{\xi}_{q}=\tilde{\mathbf{B}}_{q}(\mu) \tilde{\boldsymbol{u}},
$$

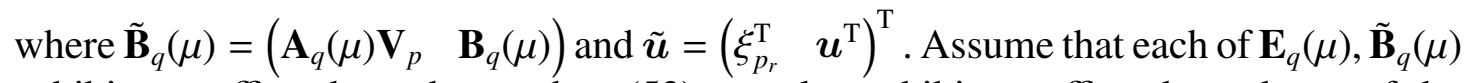
exhibits an affine dependence, then (52) can also exhibit an affine dependence of the form

$$
\left(\tilde{\mathbf{A}}_{0}+\tilde{s}_{1} \tilde{\mathbf{A}}_{1}+\ldots+\tilde{s}_{\alpha} \tilde{\mathbf{A}}_{\alpha}\right) \tilde{\xi}_{q}=\left(\tilde{\mathbf{B}}_{0}+\tilde{s}_{1} \tilde{\mathbf{B}}_{1}+\ldots+\tilde{s}_{\beta} \tilde{\mathbf{B}}_{\beta}\right) \tilde{\boldsymbol{u}}(t)
$$


where $\tilde{\mathbf{A}}_{i}$ and $\tilde{\mathbf{B}}_{i}$ are the coefficients of (52) after substituting the affine expansion (2) of each of $\mathbf{E}_{q}(\mu), \tilde{\mathbf{B}}_{q}(\mu)$. Here, the newly defined parameters $\tilde{s}_{i}, i=1, \ldots, k$, where $k=$ $\max (\alpha, \beta)$, only depends on the original parameters $\mu$. It is easy to see that the system in (53) is of the same form as the system in (46). Therefore, the algebraic state $\tilde{\xi}_{q}$ can also be written in the form (47). Hence, the projection matrix $\mathbf{V}_{q}$ can be constructed similarly as in (48). The construction of $\mathbf{V}_{q}$ for the coupling from the algebraic to the differential part can be deduced from the above discussion. Note that ROMs of systems (26) and (28) can also be derived based on the implicit moment-matching pMOR method. Since (26) and (28) are not computationally efficient, construction of the ROMs based on them will not be discussed.

It is clear, that different expansion points $\tilde{s}_{i}$ may lead to ROMs with different accuracy due to the varying dimension of the projection matrices $\mathbf{V}_{p}$ and $\mathbf{V}_{q}$ for the differential and algebraic parts, respectively. The construction of these projection matrices can be automated by adaptively selecting the expansion points using the global a posteriori error bound $\Delta(\mu, s)$ derived in [13], which is used to produce the simulation results in the next section.

For completeness, we introduce Algorithm 1 from [13] as below:

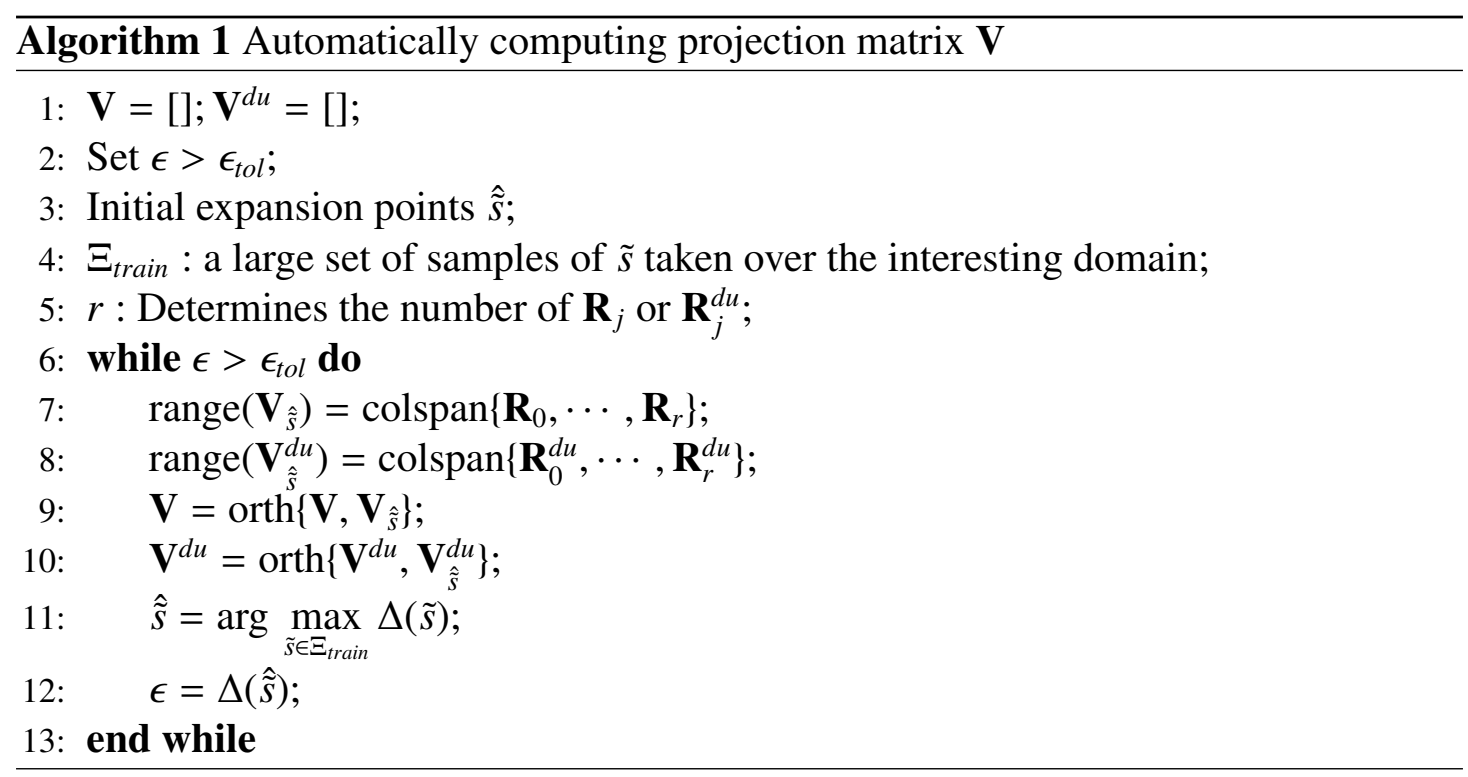

In Algorithm 1, $\mathbf{R}_{j}^{d u}$ are the vector sequences (48) computed using the matrices from the parametric dual system of (45) (see [13] for details), while $\hat{\tilde{s}}$ are the expansion points we need to select, because of (47) in order to obtain $\mathbf{V}$ automatically. Note that to implement Algorithm 1, a training set $\Xi_{\text {train }}$ of the parameter samples must be given as one of the inputs to the algorithm. They can be randomly or equidistantly selected, and should cover the whole parameter domain. 


\section{Numerical experiments}

In this section, we illustrate the robustness of the IpMOR method using coupled problems from industry. We consider electro-thermal simulation of a power-MOS device model and a package model as shown in Figure 1, originating from [14, 13].

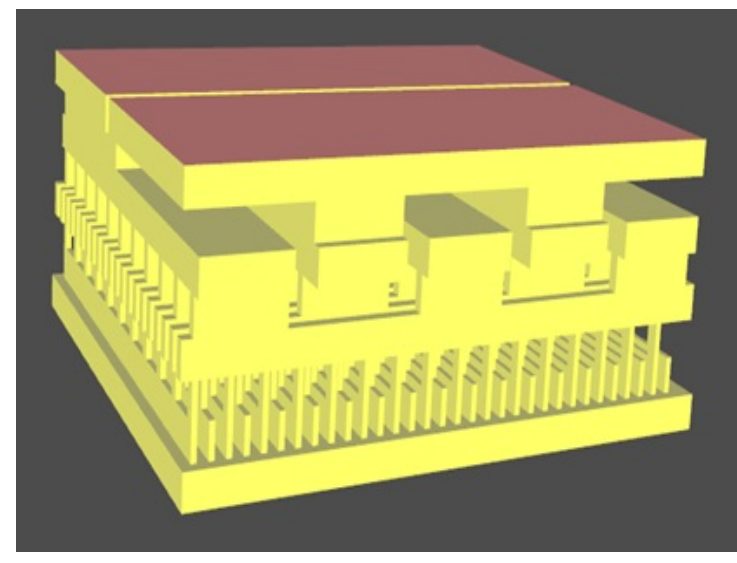

(a) A power-MOS device.

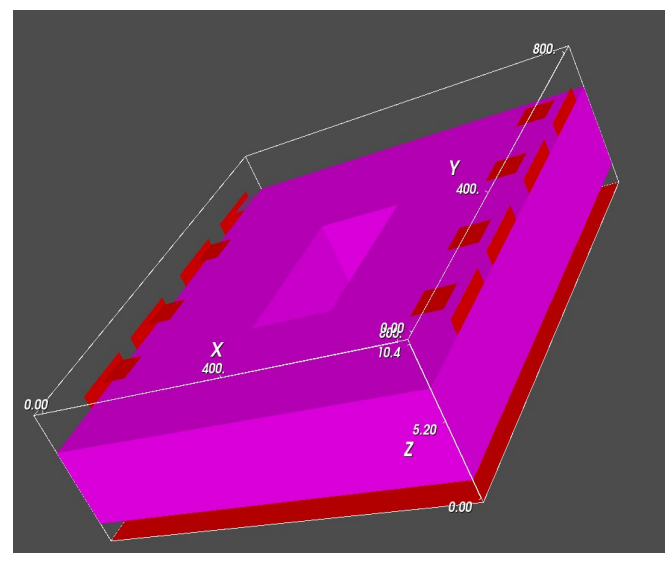

(b) A package.

Figure 1: Physical models considered in the numerical tests.

Mathematical modeling of these problems leads to the electro-thermal (ET) coupled systems in the form of (1) with system matrices

$$
\begin{aligned}
& \mathbf{E}(\mu)=\left(\begin{array}{cc}
\mathbf{0} & \mathbf{0} \\
\mathbf{0} & \mathbf{E}_{22}(\mu)
\end{array}\right), \mathbf{A}(\mu)=\left(\begin{array}{cc}
\mathbf{A}_{11}(\mu) & \mathbf{0} \\
\mathbf{0} & \mathbf{A}_{22}(\mu)
\end{array}\right), \mathbf{B}(\mu)=\left(\begin{array}{l}
\mathbf{B}_{1}(\mu) \\
\mathbf{B}_{2}(\mu)
\end{array}\right), \mathbf{C}=\left(\begin{array}{ll}
\mathbf{C}_{1}(\mu) & \mathbf{C}_{2}(\mu)
\end{array}\right), \\
& \mathcal{F}(\mu)=\left[\mathbf{0}, \ldots, \mathbf{0}, \mathbf{F}_{n_{1}+1}^{\mathrm{T}}(\mu), \ldots \mathbf{F}_{n}^{\mathrm{T}}(\mu)\right]^{\mathrm{T}} \text {, }
\end{aligned}
$$

where $\mathbf{F}_{i}=\left(\begin{array}{cc}\mathbf{F}_{11}^{i}(\mu) & \mathbf{0} \\ \mathbf{0} & \mathbf{0}\end{array}\right) \in \mathbb{R}^{n \times n}, \mathbf{F}_{11}^{i}(\mu) \in \mathbb{R}^{n_{1} \times n_{1}}, i=n_{1}+1, \ldots, n, \mathbf{E}_{22}(\mu), \mathbf{A}_{22}(\mu) \in$ $\mathbb{R}^{n_{2} \times n_{2}}, \mathbf{A}_{11}(\mu) \in \mathbb{R}^{n_{1} \times n_{1}}, \mathbf{B}_{1}(\mu) \in \mathbb{R}^{n_{1} \times m}, \mathbf{B}_{2}(\mu) \in \mathbb{R}^{n_{2} \times m}, \mathbf{C}_{1}(\mu) \in \mathbb{R}^{\ell \times n_{1}}, \mathbf{C}_{2}(\mu) \in \mathbb{R}^{\ell \times n_{2}}$. Here, $\mathbf{E}_{22}(\mu)$ and $\mathbf{A}_{11}(\mu)$ are nonsingular matrices for each $\mu$. If $\boldsymbol{x}$ is also partitioned according to (54) as $\boldsymbol{x}=\left(\boldsymbol{x}_{1}^{\mathrm{T}}, \boldsymbol{x}_{2}^{\mathrm{T}}\right)^{\mathrm{T}}$, the consistent initial condition is given by $\boldsymbol{x}(0)=$ $\left(\boldsymbol{x}_{1}(0)^{\mathrm{T}}, \boldsymbol{x}_{2}(0)^{\mathrm{T}}\right)^{\mathrm{T}}$. This system can be decoupled as follows. We observe that the index of the matrix pencil $(\mathbf{E}(\mu), \mathbf{A}(\mu))$ of (54) is independent of its nonlinearity and parameter $\mu$. Let $\mathbf{E}_{0}:=\mathbf{E}(\mu)$ and $\mathbf{A}_{0}:=\mathbf{A}(\mu)$, then we can choose the projectors

$$
\mathbf{Q}_{0}=\left(\begin{array}{ll}
\mathrm{I} & \mathbf{0} \\
\mathbf{0} & \mathbf{0}
\end{array}\right), \quad \mathbf{P}_{0}=\left(\begin{array}{ll}
\mathbf{0} & \mathbf{0} \\
\mathbf{0} & \mathrm{I}
\end{array}\right)
$$


such that $\mathbf{Q}_{0}$ is a projector onto the null space of $\mathbf{E}_{0}$, and obtain the complementary projector as $\mathbf{P}_{0}=\mathrm{I}-\mathbf{Q}_{0}$. Using (7), we obtain

$$
\mathbf{E}_{1}=\mathbf{E}_{0}-\mathbf{A}_{0} \mathbf{Q}_{0}=\left(\begin{array}{cc}
-\mathbf{A}_{11}(\mu) & \mathbf{0} \\
\mathbf{0} & \mathbf{E}_{22}(\mu)
\end{array}\right),
$$

so that $\mathbf{E}_{1}$ is uniformly nonsingular, which implies that (54) is an index-1 system. The next step is to check the type of coupling in the nonlinear part. Since $\mathcal{F}_{Q P}=\mathbf{Q}_{0}^{\mathrm{T}} \mathcal{F}(\mu) \mathbf{P}_{0}=$ $0, \mathcal{F}_{P Q}=\mathbf{P}_{0} \mathcal{F}(\mu) \mathbf{Q}_{0}=\mathbf{0}, \mathcal{F}_{P}=\mathbf{P}_{0}^{\mathrm{T}} \mathcal{F}(\mu) \mathbf{P}_{0}=\mathbf{0}$ and $\mathcal{F}_{Q}=\mathbf{Q}_{0}^{\mathrm{T}} \mathcal{F}(\mu) \mathbf{Q}_{0} \neq \mathbf{0}$, the decoupled system will take either the form (27) or (38) which is a one-way coupling from the algebraic to the differential part. Here, we only consider deriving the computationally cheaper decoupled system (38). The column matrices $\mathbf{p}_{0}$ and $\mathbf{q}_{0}$ are the linearly independent columns of the projectors $\mathbf{P}_{0}$ and $\mathbf{Q}_{0}$ in (55) given by $\mathbf{p}_{0}=\left(\begin{array}{ll}\mathbf{0} & \mathrm{I}\end{array}\right)^{\mathrm{T}}$ and $\mathbf{q}_{0}=\left(\begin{array}{ll}\mathrm{I} & \mathbf{0}\end{array}\right)^{\mathrm{T}}$, respectively. The inverse of the matrix $\left(\mathbf{p}_{0}, \mathbf{q}_{0}\right)$ is given by

$$
\left(\mathbf{p}_{0}, \mathbf{q}_{0}\right)^{-1}=\left(\begin{array}{ll}
\mathbf{0} & \mathrm{I} \\
\mathrm{I} & \mathbf{0}
\end{array}\right)=\left(\mathbf{p}_{0}^{*}, \mathbf{q}_{0}^{*}\right),
$$

where $\mathbf{p}_{0}^{* \mathrm{~T}}=\left(\begin{array}{ll}\mathbf{0} & \mathrm{I}\end{array}\right)$ and $\mathbf{q}_{0}^{* \mathrm{~T}}=\left(\begin{array}{ll}\mathrm{I} & \mathbf{0}\end{array}\right)$ are the left inverses of $\mathbf{p}_{0}$ and $\mathbf{q}_{0}$, respectively. In order to obtain the decoupled system of the form (38), we need to construct another pair of matrices $\hat{\mathbf{q}}_{0}=\left(\begin{array}{ll}\mathrm{I} & \mathbf{0}\end{array}\right)^{\mathrm{T}}$ and $\hat{\mathbf{p}}_{0}=\left(\begin{array}{ll}\mathbf{0} & \mathrm{I}\end{array}\right)^{\mathrm{T}}$, whose columns span the null spaces of $\mathbf{E}_{0}^{\mathrm{T}}$ and $\mathbf{q}_{0}^{\mathrm{T}} \mathbf{A}_{0}^{\mathrm{T}}$, respectively. Substituting the above projectors and bases into (38) and since $\mathbf{f}_{q}(\mu)=\mathbf{0}$, we obtain,

$$
\begin{aligned}
-\mathbf{A}_{11}(\mu) \xi_{q} & =\mathbf{B}_{1}(\mu) \boldsymbol{u} \\
\mathbf{E}_{22}(\mu) \xi_{p}^{\prime} & =\mathbf{A}_{22}(\mu) \xi_{p}+\xi_{q}^{\mathrm{T}} \mathbf{f}_{p q}(\mu) \xi_{q}+\mathbf{B}_{2}(\mu) \boldsymbol{u}, \quad \xi_{p}(0)=\boldsymbol{x}_{2}(0), \\
\boldsymbol{y} & =\mathbf{C}_{1}(\mu) \xi_{q}+\mathbf{C}_{2}(\mu) \xi_{p}+\mathbf{D}(\mu) \boldsymbol{u}
\end{aligned}
$$

where $\mathbf{f}_{p q}(\mu)=\left[\mathbf{F}_{11}^{n_{1}+1^{\mathrm{T}}}(\mu), \ldots, \mathbf{F}_{11}^{n^{\mathrm{T}}}(\mu)\right]^{\mathrm{T}} \in \mathbb{R}^{n_{1} \times n_{1} \times n_{2}}$. From (56) we see that the system of quadratic DAEs (54) is decoupled into $n_{q}=n_{1}$ algebraic equations (56a) and $n_{p}=n_{2}$ differential equations (56b), with $n=n_{1}+n_{2}$. Here, the initial condition $\boldsymbol{x}_{1}(0)=\xi_{q}(0)$ must satisfy the hidden constraint $\xi_{q}(0)=-\mathbf{A}_{11}(\mu)^{-1} \mathbf{B}_{1}(\mu) \boldsymbol{u}(0)$ and $\boldsymbol{x}_{2}(0)=\xi_{p}(0)$ can be arbitrarily chosen. We can now apply the proposed IpMOR method for quadratic DAEs to (56) instead of (54).This is illustrated in Examples 7.1 and 7.2, for the case of the device and the package models, respectively.

Remark 1. Note that with the special structure of (54), the one-way decoupled system in (56) can be directly obtained by inserting the partition of $\boldsymbol{x}=\left(\xi_{q}^{\mathrm{T}}, \xi_{p}^{\mathrm{T}}\right)^{\mathrm{T}}$ into (54). The above analysis shows a formal derivation of (56) without taking advantage of the special form of (54). In particular, if the structure of the tensor $\mathcal{F}$ is unclear, then derivation of (56) cannot be straightforward. 
All simulations were done using in MATLAB $₫$ Version 2012 b on a Laptop with 6GB RAM, CPU@ $2.00 \mathrm{GHz}$. The sparse tensor computations were performed with the MATLAB Tensor Toolbox [15, 16]. All used methods are defined as follows:

(a) We use D-pMOR to denote the method directly applying the existing pMOR based on multi-moment-matching (Algorithm 1) to the original system of quadratic DAEs in (54).

(b) IpMOR is the proposed method in Section 5, where pMOR using Algorithm 1 is applied to the subsystems (56a) and (56b) separately.

(c) For the power-MOS device model, we will see from Remark 2 that the differential subsystem (56b) can be approximately considered as a non-parametric system, so that MOR based on moment-matching, such as PRIMA [17], rather than pMOR based on multi-moment-matching, can be applied to construct the ROM of (56b). Therefore, for this special structure, we apply pMOR (Algorithm 1) to (56a), and apply PRIMA to (56b). We call this method IpMOR-PRIMA.

Example 7.1. In this example, we consider a power-MOS device model illustrated in Figure 1a. It is a parameterized quadratic system described in (1) with matrices and tensor in the form of (54) and $n=13216$ state variables. There are $m=6$ and $\ell=$ 12 inputs and outputs, respectively. The matrices $\mathbf{E}(\mu), \mathbf{A}(\mu), \mathbf{B}(\mu), \mathbf{C}(\mu), \mathbf{D}(\mu)$ and the tensor $\mathcal{F}(\mu)$ exhibit a parameter dependence of an affine form given by

$$
\mathbf{M}(\mu)=\mathbf{M}_{0}+\mu \mathbf{M}_{1},
$$

where $\mathbf{M}(\mu)$ indicates any parametrized matrix or tensor in (1) and $\mathbf{M}_{i}$ are parameterindependent coefficients. For this example, $\mu=\sigma$ describes the electrical conductivity of the selected materials of the power-MOS device. The system is excited by the input $\boldsymbol{u}(t)=\left(\boldsymbol{u}_{1}(t), \ldots, \boldsymbol{u}_{6}(t)\right)^{\mathrm{T}}$, where $\boldsymbol{u}_{1}(t)=\boldsymbol{u}_{3}(t)=\mathbf{0}, \boldsymbol{u}_{4}(t)=\boldsymbol{u}_{5}(t)=\boldsymbol{u}_{6}(t)=300$ and $\boldsymbol{u}_{2}(t)=\left\{\begin{array}{ll}10^{7} t, & \text { if } 0 \leq t \leq 10^{-6}, \\ 10, & \text { Otherwise. }\end{array}\right.$ This system is decoupled into the form (56) with $n_{p}=n_{1}=11556$ differential equations, and $n_{q}=n_{2}=1660$ algebraic equations. The initial condition for the algebraic subsystem has to satisfy (56a) at $t=0$ and the initial condition for the differential system is 300 for all variables since it can be arbitrarily chosen. We intend to reduce the decoupled power-MOS model using the proposed IpMOR method combined with Algorithm 1, so that the projection matrices $\mathbf{V}_{q} \in \mathbb{R}^{n_{q} \times r_{1}}$ and $\mathbf{V}_{p} \in \mathbb{R}^{n_{p} \times r_{2}}$ are automatically constructed for (56a) and (56b), respectively. To construct $\mathbf{V}_{q}$ we use a training set of 100 randomly distributed parameter samples ( $\tilde{s}=$ $\sigma)$ in the interval $\sigma \in\left[10^{6}, 10^{7}\right]$ for Algorithm 1. To construct $\mathbf{V}_{p}$, we pair 10 randomly distributed samples of frequency $f$ with 10 random samples of parameter $\sigma \in\left[10^{6}, 10^{7}\right]$ to form the training set. Then samples of $s$ are obtained from the relation $s=2 \pi \mathrm{J}$, 
where $J=\sqrt{-1}(\tilde{s}=(\sigma, s))$. In addition, we use $r=2$ and $r=4$ in (49) to construct $\mathbf{V}_{q}$ and $\mathbf{V}_{p}$, respectively.

Table 1: Convergence behavior of Algorithm 1 using IpMOR.

\begin{tabular}{ccccccc}
\hline & \multicolumn{3}{c}{ Algebraic subsystem, $n_{q}=1660, \epsilon_{\text {tol }}=10^{-12}$} & \multicolumn{3}{c}{ Differential subsystem, $n_{p}=11556, \epsilon_{\text {tol }}=10^{-6}$} \\
\hline Iterations & Selected sample $\hat{\sigma}$ & Error bound & $r_{1}$ & Selected pair $(\hat{\sigma}, \hat{s})$ & Error bound & $r_{2}$ \\
\hline 1 & $1.3396 \times 10^{6}$ & $9.70 \times 10^{-10}$ & 2 & $\left(9.1263 \times 10^{6}, 4.9450 \times 10^{7} \mathrm{~J}\right)$ & $2.68 \times 10^{2}$ & 6 \\
2 & $9.9917 \times 10^{6}$ & $6.28 \times 10^{-12}$ & 4 & $\left(1.7314 \times 10^{6}, 2.0427 \times 10^{7} \mathrm{~J}\right)$ & $2.59 \times 10^{1}$ & 12 \\
3 & - & - & - & $\left(8.5282 \times 10^{6}, 3.5865 \times 10^{7} \mathrm{~J}\right)$ & $2.88 \times 10^{0}$ & 18 \\
4 & - & - & - & $\left(9.1829 \times 10^{6}, 5.7256 \times 10^{7} \mathrm{~J}\right)$ & $2.08 \times 10^{-1}$ & 24 \\
5 & - & - & - & $\left(1.7314 \times 10^{6}, 2.9001 \times 10^{7} \mathrm{~J}\right)$ & $2.52 \times 10^{-3}$ & 30 \\
6 & - & - & - & $\left(1.7314 \times 10^{6}, 5.4763 \times 10^{7} \mathrm{~J}\right)$ & $1.93 \times 10^{-4}$ & 34 \\
7 & - & - & - & $\left(3.7886 \times 10^{6}, 2.9760 \times 10^{7} \mathrm{~J}\right)$ & $1.03 \times 10^{-5}$ & 38 \\
8 & - & - & - & $\left(9.1263 \times 10^{6}, 3.7540 \times 10^{7} \mathrm{~J}\right)$ & $2.76 \times 10^{-8}$ & 42 \\
\hline
\end{tabular}

Table 1 shows the selected expansion point: $(\hat{\tilde{s}}=\hat{\sigma})$ or $(\hat{\tilde{s}}=(\hat{\sigma}, \hat{s}))$, the calculated output error bound and the number of columns $\left(r_{1}, r_{2}\right)$ of the projection matrices for each iteration. Algorithm 1 terminates after 2 and 8 iterations with the reduced order of 4 and 42 for the algebraic and differential subsystems, respectively. Thus using the proposed IpMOR method, the algebraic subsystem is reduced from order 1160 to order 4 , and the differential subsystem is reduced from order 11556 to order 42 . Hence the power-MOS model is reduced from order 13216 to order 46.

Remark 2. We note that, in general, the parameter dependence of the power-MOS device model takes the form (57). However, after being decoupled into the one-way coupled system (56), we observe that the system matrices of the differential subsystem are of the form $\mathbf{M}(\sigma)=\mathbf{M}_{0}+\sigma \mathbf{M}_{1}, \quad\left(\mathbf{M}=\mathbf{E}_{22}, \mathbf{A}_{22}, \mathbf{B}_{2}, \mathbf{C}_{2}\right)$, with $\left[\mathbf{M}_{1}\right]_{i j}<\epsilon_{\text {mach }}$, where $\epsilon_{\text {mach }}$ is the machine precision. Therefore, the parameter part $\sigma \mathbf{M}_{1}$ can be ignored, so that $\mathbf{M}(\sigma) \approx \mathbf{M}_{0}$ for the differential subsystem. This implies that we can apply Algorithm 1 to the algebraic subsystem and conventional MOR such as PRIMA [17] to reduce the non-parametric differential subsystem, which constitutes the IpMORPRIMA method mentioned before. We construct $\mathbf{V}_{q}$ as before and construct $\mathbf{V}_{p}$ using PRIMA by matching 10 moments associated with the expansion point at $f=10^{2}$ [17]. We were able to reduce the algebraic subsystem from order 1160 to order 4 , and the differential subsystem from order 11556 to order 44, respectively. This leads to a pROM of order 48 using the IpMOR method.

For comparison, we also use D-pMOR to construct the ROM of (54), i.e., we apply Algorithm 1 to the linear part of (54) with $\mathbf{M}(\sigma)=\mathbf{M}_{0}+\sigma \mathbf{M}_{1}, \quad(\mathbf{M}=\mathbf{E}, \mathbf{A}, \mathbf{B}, \mathcal{F}, \mathbf{C}, \mathbf{D})$, using the same training set used by the IpMOR method for the differential subsystem. Table 2 shows the selected expansion points, calculated output error bound and the number of columns of the projection matrix $\mathbf{V} \in \mathbb{R}^{n \times r}$ at each iteration. We observe that the 
algorithm terminates after 8 iterations with $r=48$ columns of $\mathbf{V}$ at an accuracy of $\epsilon_{\text {tol }}=10^{-8}$. Hence the power-MOS device model is reduced from 13216 to 48, using D-pMOR.

Table 2: Convergence behavior of Algorithm 1 using D-pMOR.

\begin{tabular}{cccc}
\hline \multicolumn{4}{c}{$\mathrm{n}=13216, \epsilon_{\text {tol }}=10^{-6}}$. \\
\hline Iterations & Selected samples $\left(\sigma_{0}, s_{0}\right)$ & Error bound & $r$ \\
\hline 1 & $\left(9.1263 \times 10^{6}, 4.9450 \times 10^{7} \mathrm{~J}\right)$ & $2.68 \times 10^{2}$ & 8 \\
2 & $\left(9.1263 \times 10^{6}, 2.0427 \times 10^{7} \mathrm{~J}\right)$ & $3.32 \times 10^{1}$ & 14 \\
3 & $\left(9.8768 \times 10^{6}, 5.7256 \times 10^{7} \mathrm{~J}\right)$ & $4.34 \times 10^{0}$ & 21 \\
4 & $\left(1.9860 \times 10^{6}, 2.9001 \times 10^{7} \mathrm{~J}\right)$ & $1.59 \times 10^{-1}$ & 29 \\
5 & $\left(1.2646 \times 10^{6}, 3.7540 \times 10^{7} \mathrm{~J}\right)$ & $2.95 \times 10^{-3}$ & 35 \\
6 & $\left(9.8768 \times 10^{6}, 5.4763 \times 10^{7} \mathrm{~J}\right)$ & $2.40 \times 10^{-4}$ & 39 \\
7 & $\left(3.7886 \times 10^{6}, 2.9760 \times 10^{7} \mathrm{~J}\right)$ & $9.02 \times 10^{-6}$ & 44 \\
8 & $\left(9.1263 \times 10^{6}, 3.5865 \times 10^{7} \mathrm{~J}\right)$ & $3.34 \times 10^{-8}$ & 48 \\
\hline
\end{tabular}

Table 3: Simulation efficiency comparison.

\begin{tabular}{lccccccc}
\hline \multicolumn{7}{c}{ Original model } & Reduced order \\
\hline pMOR methods & $\mathrm{n}$ & $m$ & $\ell$ & $\mathrm{r}$ & Speed-up & Reduction rate (\%) & Error \\
\hline D-pMOR & 13,216 & 6 & 12 & 48 & 538.9 & 99.6 & $7.5 \times 10^{-2}$ \\
IpMOR & 13,216 & 6 & 12 & 46 & 2116.5 & 99.7 & $4.1 \times 10^{-3}$ \\
IpMOR-PRIMA & 13,216 & 6 & 12 & 48 & 1288.3 & 99.6 & $5.1 \times 10^{-3}$ \\
\hline
\end{tabular}

Next, we simulate all the above three ROMs within the time interval $t \in\left[0,10^{-6}\right]$ and the electrical conductivity $\sigma \in\left[10^{6}, 10^{8}\right]$. We discretized the time and the electrical conductivity into 100 and 50 grid points, respectively, using the implicit Euler integration method for the differential subsystem. The efficiency comparison of these ROMs are summarized in Table 3 and the error is defined as

$$
\max _{\mu \in\left[10^{6}, 10^{10}\right]} \max _{j \in\{1, \ldots, \ell\}}\left\|y_{j}(\mu)-y_{j_{r}}(\mu)\right\|_{2} /\left\|y_{j}(\mu)\right\|_{2},
$$

where $y_{j}(\mu)$ and $y_{j_{r}}(\mu)$ are the outputs of the original large model and ROM, respectively at port $j$ depending on the electrical conductivity $\mu=\sigma$ of the power-MOS device. They are vectors containing the output values at $t_{i}, i=1, \ldots, \mathrm{N}$ uniform time instances in the time interval $\left[0,10^{-6} s\right]$.

It is seen that the ROMs derived by IpMOR and IpMOR-PRIMA are much faster to be simulated and more accurate than is ROM derived from D-pMOR. Figure 3 shows the comparison of the thermal flux density and its relative error at port 9 for each method. 


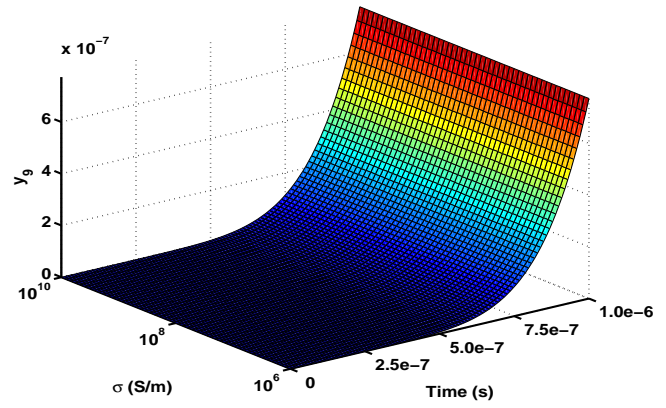

(a) Thermal flux at port $9, \boldsymbol{y}_{9}$.

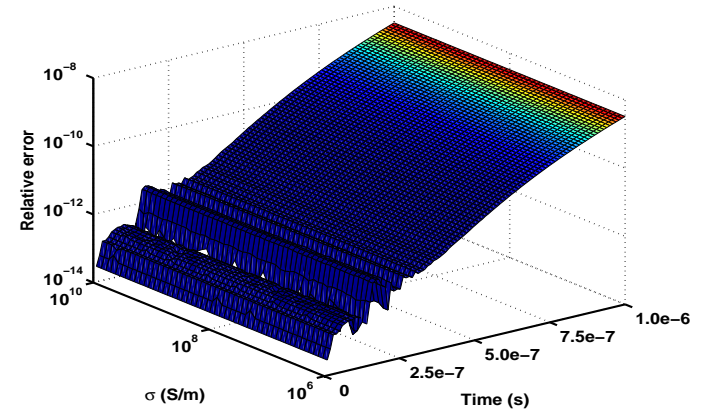

(c) Relative error of IpMOR.

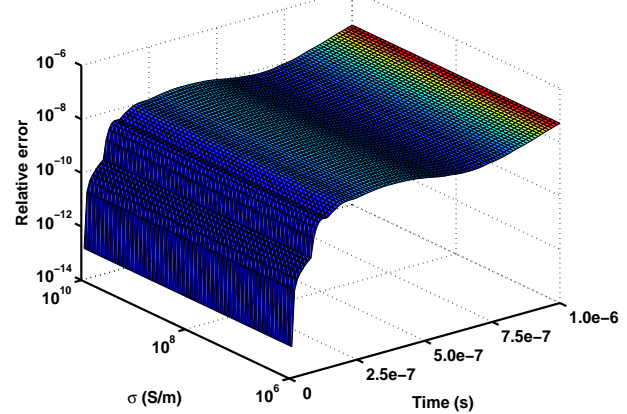

(b) Relative error of D-pMOR.

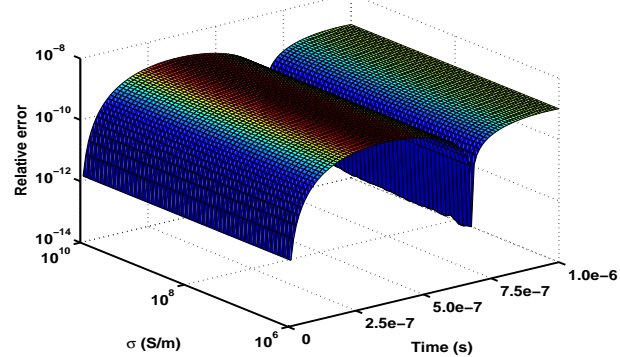

(d) Relative error of the IpMOR-PRIMA.

Figure 2: Comparison of thermal flux and its relative error at port 9.

Example 7.2. We consider a package model [18] illustrated in Figure 1b. It is also a system with matrices and tensor of the form (54) with dimension $n=9193$. This system has $m=34$ and $\ell=68$ inputs and outputs, respectively. The system matrices and tensor exhibit affine parameter dependence $\mathbf{M}(h)=\mathbf{M}_{0}+h \mathbf{M}_{1}+\frac{1}{h} \mathbf{M}_{2}$, where $\mathbf{M}(h)$ is any parametrized matrix or tensor in (1) and $\mathbf{M}_{i}$ are parameter-independent coefficients. For this example, $h$ represents the different thicknesses of the top layer of the package. The system is excited by the input $\boldsymbol{u}(t)=\left(\boldsymbol{u}_{1}(t), \ldots, \boldsymbol{u}_{34}(t)\right)^{\mathrm{T}}$, where $\boldsymbol{u}_{1}(t)=1, \boldsymbol{u}_{2}(t)=\cdots=\boldsymbol{u}_{17}(t)=0$, and $\boldsymbol{u}_{19}(t)=\cdots=\boldsymbol{u}_{34}(t)=348.15$, $\boldsymbol{u}_{18}(t)= \begin{cases}75 \times 10^{8} t+348.15, & \text { if } t \leq 10^{-8}, \\ 423.15, & \text { if } t>10^{-8} .\end{cases}$

The initial condition for the algebraic subsystem has to satisfy (56a) at $t=0$ and the initial condition for the differential system is 348.15 for all variables since it can arbitrarily chosen. This system can also be decoupled into a one-way coupled system of the form (56) with $n_{p}=n_{1}=8071$ differential and $n_{q}=n_{2}=1122$ algebraic equations. The projection matrices $\mathbf{V}_{q} \in \mathbb{R}^{n_{q} \times r_{1}}$ and $\mathbf{V}_{p} \in \mathbb{R}^{n_{p} \times r_{2}}$ are constructed by the IpMOR method with Algorithm 1 for (56a) and (56b), respectively. To construct $\mathbf{V}_{q}$ we use 100 randomly 
distributed parameter samples $(\tilde{s}=h)$ of $h \in(0,100]$ as training set for Algorithm 1 . To construct $\mathbf{V}_{p}$, we pair 5 randomly distributed samples of $s=2 \pi J f, f \in\left[0,10^{4}\right]$ with 10 random samples of the parameter $h \in(0,100]$ to form the training set of Algorithm 1 $(\tilde{s}=(h, s))$. We used $r=4$ and $r=2$ in (49) to construct $\mathbf{V}_{q}$ and $\mathbf{V}_{p}$, respectively.

Table 4: Convergence behavior of Algorithm 1 using IpMOR, $\epsilon_{t o l}=10^{-5}$.

\begin{tabular}{ccccccc}
\hline & \multicolumn{3}{c}{ Algebraic subsystem, $n_{q}=1122$} & \multicolumn{3}{c}{ Differential subsystem, $n_{p}=8071$} \\
\hline Iterations & Selected sample $\hat{h}$ & Error bound & $r_{1}$ & Selected pair $(\hat{h}, \hat{s})$ & Error bound & $r_{2}$ \\
\hline 1 & 99.9743 & $3.86 \times 10^{-9}$ & 24 & $\left(77.1617,1.7189 \times 10^{4} \mathrm{~J}\right)$ & $4.07 \times 10^{1}$ & 98 \\
2 & - & - & - & $\left(5.3648,5.7132 \times 10^{4} \mathrm{~J}\right)$ & $5.37 \times 10^{-1}$ & 196 \\
3 & - & - & - & $\left(48.757,5.7132 \times 10^{4} \mathrm{~J}\right)$ & $2.39 \times 10^{-2}$ & 294 \\
4 & - & - & - & $\left(8.9365,5.7132 \times 10^{4} \mathrm{~J}\right)$ & $1.87 \times 10^{-3}$ & 392 \\
5 & - & - & - & $\left(5.3648,4.1597 \times 10^{4} \mathrm{~J}\right)$ & $3.36 \times 10^{-4}$ & 490 \\
6 & - & - & - & $\left(77.1617,5.7132 \times 10^{4} \mathrm{~J}\right)$ & $5.02 \times 10^{-5}$ & 588 \\
7 & - & - & - & $\left(5.3648,1.7189 \times 10^{4} \mathrm{~J}\right)$ & $2.60 \times 10^{-6}$ & 675 \\
\hline
\end{tabular}

Table 4 shows the selected expansion point, the calculated output error bound and the number of columns $\left(r_{1}, r_{2}\right)$ of the projection matrices for each iteration. Algorithm 1 terminates after 1 and 7 iterations with the reduced order of 24 and 675 for the algebraic and differential subsystems, respectively. Thus using the proposed adaptive IpMOR method, the algebraic subsystem is reduced from order 1122 to order 24 , and the differential subsystem is reduced from order 8071 to order 675 . Hence the package model is reduced from order 9193 to order 699. For comparison, we apply D-pMOR to the linear part of the quadratic DAEs in (54), by making use of the same training set which we applied for the IpMOR method on the differential subsystem. Table 5 shows the selected expansion point, calculated output error bound and the number of columns of the projection matrix $\mathbf{V} \in \mathbb{R}^{n \times r}$ at each iteration. The algorithm terminates after 6 iterations with $r=48$ number of columns of $\mathbf{V}$. Hence this model is reduced from 9193 to 719 .

Table 5: Convergence behavior of Algorithm 1 using D-pMOR.

\begin{tabular}{cccc}
\hline \multicolumn{4}{c}{$\mathrm{n}=9193, \epsilon_{\text {tol }}=10^{-5}$} \\
\hline Iterations & Selected samples $\left(h_{0}, s_{0}\right)$ & Error bound & $r$ \\
\hline 1 & $\left(77.1617,1.7189 \times 10^{4} \mathrm{~J}\right)$ & $5.49 \times 10^{-1}$ & 124 \\
2 & $\left(5.3648,5.7132 \times 10^{4} \mathrm{~J}\right)$ & $1.60 \times 10^{-2}$ & 248 \\
3 & $\left(77.1617,5.7132 \times 10^{4} \mathrm{~J}\right)$ & $5.69 \times 10^{-3}$ & 372 \\
4 & $\left(48.757,5.7132 \times 10^{4} \mathrm{~J}\right)$ & $6.34 \times 10^{-4}$ & 496 \\
5 & $\left(8.9365,5.7132 \times 10^{4} \mathrm{~J}\right)$ & $2.94 \times 10^{-5}$ & 619 \\
6 & $\left(5.3648,4.1597 \times 10^{4} \mathrm{~J}\right)$ & $2.94 \times 10^{-6}$ & 719 \\
\hline
\end{tabular}


Table 6: Simulation efficiency comparison.

\begin{tabular}{lccccccc}
\hline \multicolumn{7}{c}{ Original model } & \multicolumn{2}{c}{ Reduced order } \\
\hline pMOR methods & $\mathrm{n}$ & $m$ & $\ell$ & $\mathrm{r}$ & Speed-up & Reduction rate (\%) & Error \\
\hline D-pMOR & 9193 & 34 & 68 & 719 & - & 92.2 & - \\
IpMOR & 9193 & 34 & 68 & 699 & 6.4 & 92.4 & $7.7 \times 10^{-3}$ \\
\hline
\end{tabular}

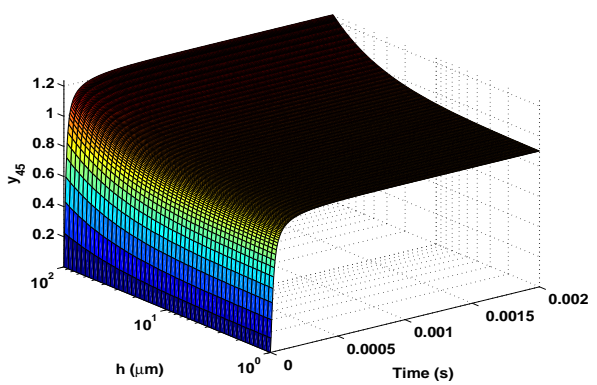

(a) Output solution of the IpROM.

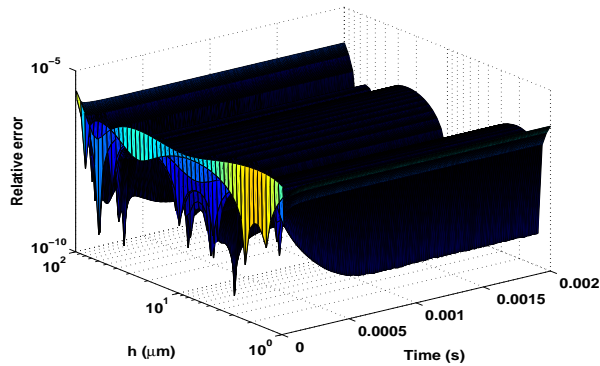

(b) Relative error of the outputs.

Figure 3: Output solution and relative error of the IpMOR for the package model.

We tried to simulate both parametric ROMs in the time interval $t \in\left[0,2 \times 10^{-3} s\right]$ and thickness interval $h \in\left(0,10^{2}\right]$, using the implicit Euler integration method on the same grid points. It is observed that it is impossible to simulate the ROM constructed by D-pMOR while the ROM derived by IpMOR is easy to be simulated, and results in a speed-up of 6.4 as shown in Table 6. This is due to that fact that applying D-pMOR to the system of quadratic DAEs leads to a ROM of ODEs which behaves like a system of DAEs with higher index. In Table 6, we used the same error definition as in (58) with $\mu=h$ in the time interval $t \in\left[0,2 \times 10^{-3}\right]$. Figure 3 shows the thermal flux density produced by IpMOR, and its relative error at port 45 at different thicknesses of the top layer, indicating that the proposed IpMOR method is sufficiently accurate.

\section{Conclusions}

We have proposed an index-aware parametric model order reduction (IpMOR) method, and have illustrated its superiority over the existing approaches with examples from industry. We have observed that IpMOR is computationally cheaper and more accurate than directly applying pMOR to the original coupled system, and it always preserves the index-structure of the original quadratic DAEs. This technique accelerates uncertainty quantification and simulation of quadratic parameterized DAEs. Note that the IpMOR method is independent of the choice of the existing pMOR method used to reduced the subsystems. However, for more general structured DAEs, one has to be aware that the 
numerical computation of the bases for the decoupling may involve serious difficulties because of the accuracy sensitive rank decisions. Finally, our method could be extended to more complicated quadratic DAEs whose index always depends on the nonlinearity, and those DAEs with higher index. This could be a topic in the future.

\section{Acknowledgements}

This work was supported by the collaborative project nanoCOPS, Nanoelectronics COupled Problems Solutions, supported by the European Union in the FP7-ICT-201311 Program under Grant Agreement Number 619166.

\section{References}

[1] P. Benner, S. Gugercin, K. Willcox, A survey of model reduction methods for parametric systems, SIAM Review 57 (4) (2015) 483-531.

[2] U. Baur, P. Benner, L. Feng, Model Order Reduction for Linear and Nonlinear Systems: A System-Theoretic Perspective, Arch. Comput. Methods Eng. 21 (4) (2014) 331-358. doi:10.1007/s11831-014-9111-2.

[3] N. Banagaaya, Index-aware model order reduction methods, Ph.D. thesis, Eindhoven University of Technology, Eindhoven, Netherlands (2014).

[4] N. Banagaaya, G. Alì, W. Schilders, Index-aware Model Order Reduction Methods: Applications to Differential-Algebraic Equations, Vol. 2 of Atlantis Studies in Scientific Computing in Electromagnetics, Atlantis Press, 2016.

[5] V. Mehrmann, T. Stykel, Balanced truncation model reduction for large-scale systems in descriptor form, in: P. Benner, V. Mehrmann, D. C. Sorensen (Eds.), Dimension Reduction of Large-Scale Systems, Vol. 45 of Lect. Notes Comput. Sci. Eng., Springer-Verlag, Berlin/Heidelberg, Germany, 2005, pp. 83-115.

[6] P. Benner, T. Stykel, Model order reduction for differential-algebraic equations: A survey, in: A. Ilchmann, T. Reis (Eds.), Surveys in Differential-Algebraic Equations IV, Differential-Algebraic Equations Forum, Springer International Publishing, 2017, pp. 107-160. doi:10.1007/978-3-319-46618-7_3.

[7] G. Alì, N. Banagaaya, W. Schilders, C. Tischendorf, Index-aware model order reduction for differential-algebraic equations, Math. Comput. Model. Dyn. Syst. 20 (4) (2013) 345-373.

[8] R. März, The index of linear differential-algebraic equations with properly stated leading terms, Results in Mathematics 42 (3) (2002) 308-338. 
[9] N. Banagaaya, G. Alì, W. Schilders, Implicit-IMOR method for index-1 and index-2 linear constant DAEs, external Report, CASA Report, No. 14-10. Eindhoven: Technische Universiteit Eindhoven, 28 pp., 2014, Available from http://www. win. tue.nl/analysis/reports/rana14-10.pdf.

[10] R. März, Canonical projectors for linear differential algebaric equations, Computers Math. Applications 31 (4/5) (1996) 121-135.

[11] L. Feng, P. Benner, Reduced Order Methods for modeling and computational reduction, MS\&A Series, Vol. 9, Springer-Verlag, Berlin, Heidelberg, New York, 2014, Ch. 6: A robust algorithm for parametric model order reduction based on implicit moment matching, pp. 159-186.

[12] F. Chen, J. Hesthaven, X. Zhu, On the use of reduced basis methods to accelerate and stabilize the parareal method, in: Reduced Order Methods for Modeling and Computational Reduction, MS\&A Series A. Quarteroni, G. Rozza (editors), Vol. 9, Springer, 2014, pp. 159-186.

[13] L. Feng, Y. Yue, N. Banagaaya, P. Meuris, W. Schoenmaker, P. Benner, Parametric modeling and model order reduction for (electro-)thermal analysis of nanoelectronic structures, Mathematics in Industry 6 (10) (2016) 1-16.

[14] N. Banagaaya, L. Feng, W. Schoenmaker, P. Meuris, A. Wieers, R. Gillon, P. Benner, Model order reduction for nanoelectronics coupled problems with many inputs, in: In: Proceedings 2016 Design, Automation \& Test in Europe Conference \& Exhibition (DATE 2016), March 14-16, IEEE, 2016, pp. 313-318.

[15] B. W. Bader, T. G. Kolda, et al., MATLAB Tensor Toolbox Version 2.6, Available online (February 2015).

URL http://www . sandia.gov/ tgkolda/TensorToolbox/

[16] B. W. Bader, T. G. Kolda, Efficient MATLAB computations with sparse and factored tensors, SIAM J. Sci. Comput. 30 (1) (2007) 205-231.

[17] A. Odabasioglu, M. Celik, L. T. Pileggi, PRIMA: passive reduced-order interconnect macromodeling algorithm, IEEE Trans. Comput.-Aided Design Integr. Circuits Syst. 17 (8) (1998) 645-654.

[18] N. Banagaaya, L. Feng, P. Meuris, W. Schoenmaker, P. Benner, Model order reduction of an electro-thermal package model, FAC-PapersOnLine. 8 (1) (2015) 934-935. 